BULLETIN Bulletin hispanique

HISPANIQUE Université Michel de Montaigne Bordeaux

116-1 | 2014

Varia

\title{
Théories musicales
}

entre incantations et prières

Daniel Grégorio

\section{(2) OpenEdition}

Journals

Édition électronique

URL : http://journals.openedition.org/bulletinhispanique/2990

DOI : 10.4000/bulletinhispanique.2990

ISSN : 1775-3821

Éditeur

Presses universitaires de Bordeaux

Édition imprimée

Date de publication : 1 juin 2014

Pagination : 283-318

ISBN : 978-2-86781-931-5

ISSN : 0007-4640

Référence électronique

Daniel Grégorio, « Théories musicales », Bulletin hispanique [En ligne], 116-1 | 2014, mis en ligne le 01 juin 2017, consulté le 26 mai 2020. URL : http://journals.openedition.org/bulletinhispanique/2990 ; DOI : https://doi.org/10.4000/bulletinhispanique.2990 


\title{
VARIÉTÉS
}

\section{Théories musicales : entre incantations et prières}

\author{
DANIEL GRÉgorio \\ CALHISTE - Université de Valenciennes
}

L'opération magique impose le respect du rituel dans la gestuelle et les enchantements. Néanmoins, les grimoires n'expliquent pas les modalités d'énonciation des paroles magiques. Seules les théories musicales peuvent apporter une information complémentaire sur cet aspect des pratiques magiques. Exception faite de la place de l'esthétisme dans le chant, surtout liturgique, qui n'est pas abordé par les grimoires, les différents éléments qui constituent la théorie musicale conviennent à la réalisation des enchantements.

Mots-clés : musique, magie, incantation, proportions, rythmes.

El ritual mágico impone el estricto respeto en la realización de los gestos y de los encantamientos. Sin embargo los grimorios no ofrecen ninguna información sobre el modo de enunciación de las palabras mágicas. Al tratarse de un discurso oral modulado, sólo las teorías musicales pueden aportar información sobre este aspecto de las prácticas mágicas. Exceptuando la reflexión sobre la estética del canto litúrgico, que no aparece en ningún grimorio, los diferentes elementos que pueden conformar la teoría musical, pueden convenir a la realización de los encantamientos.

Palabras Claves: Música, magia, incantación, proporciones, ritmo.

Magic demands that ritual should be respected for movements and enchantments. Although books of spells explain the steps to achieve a magic ritual, they set no details on a specific pronunciation of magic words. Musical theories only can bring additional information on this aspect of magic practices. Except the problem of aestheticism in singing, mainly in liturgical singing which is not mentioned in magic books, the diverse elements of the musical theory may fit, for the most part, the spell ritual.

Keywords : Music, magic, incantation, proportions, rhythm. 
Celon un dicton espagnol, la musique apaise les fauves. Les bêtes seraient $\checkmark$ ainsi sensibles aux charmes de la mélodie. Cette dernière, perçue comme une création spécifiquement humaine, ne ferait que manifester le pouvoir de l'homme sur des êtres considérés comme inférieurs. Cette supériorité est établie dans la Bible lorsque Adam nomme les animaux, se plaçant ainsi, par volonté divine, au sommet de la Création ${ }^{1}$, elle-même créée par l'énonciation du Verbe $^{2}$. Musique et parole semblent pouvoir dominer des créatures considérées comme inférieures, d'un point de vue spirituel ou intellectuel, sans pour autant dévoiler le rapport qui peut exister entre elles.

Cette faculté est également reprise par divers récits mythologiques qui proposent néanmoins des variantes substantielles. Cette fois, ce ne sont plus les animaux qui sont subjugués par le pouvoir des sons, mais les hommes. Le chant des sirènes cause la perte des marins téméraires qui ne suivent pas les conseils d'une grande magicienne comme Circé ou qui ne sont pas sous la protection d'un musicien comme Orphée.

Lorsqu'on étudiela magie médiévale, dans sa représentation historiographique et mythologique, on peut remarquer les liens qui existent entre le mage et le musicien $^{3}$. De même, les indications contenues dans les grimoires présentent souvent des rituels spécifiques pour la réalisation d'enchantements qui, par la répétition de certains mots, altèrent le monde et dominent la volonté des hommes.

Le musicien devient alors un être à part : il possèderait un pouvoir incommensurable qui lui permettrait, de dominer la nature et les hommes, voire de franchir les frontières qui séparent l'humain du divin ${ }^{4}$. Or, ce pouvoir attribué aux paroles énoncées et aux mélodies produites, n'est pas un topos exclusivement mythologique ou légendaire. En 572, le concile de Braga II interdit de réaliser le tissage des robes ${ }^{5}$ et la cueillette de plantes médicinales ${ }^{6}$ au rythme d'incantations qui ne seraient pas des prières à Dieu. La notion d'incantation introduit un élément supplémentaire qui ne se réfère pas au contenu, ou au sens exact des paroles prononcées, mais à la modalité de leur énonciation. Le terme incantare renvoie à l'idée de chant, de réalisation dans le chant (in-cantare, en-chanter/en-cantar) ou par le chant (ex-cantare). Les dispositions conciliaires démontrent que l'Antiquité et le Moyen Âge croient " de toute évidence à l'efficacité puissante que possèdent certains rites vocaux, les carmina ${ }^{7}$, dont la valeur positive ou négative peut provenir aussi bien de leur contenu que du contexte dans lequel ils sont entonnés.

1. Genèse, II, 19-20.

2. Genèse, I, 1, 3.

3. Daniel Grégorio, «Du mythe à la pratique, musique et magie dans l'œuvre d'Alphonse X», Bulletin Hispanique, 112, 2010, p. 743-762.

4. Orfeo, Argonáuticas, edición de Miguel Periago Lorente, Madrid, 1987, p. 67-68.

5. José Vives, Concilios visigóticos e hispano-romanos, Madrid, 1963, Concile de Braga II, Canon LXXV, p. 104.

6. Ibid., Canon LXXIV, p. 103.

7. Fritz Graf, La magie dans l'antiquité gréco-romaine, Paris, 2004, p. 53. 
Les canons conciliaires, autant que les écrits magiques et les récits mythologiques, mettent en exergue le rôle de la parole dans l'opération magique. Cependant, bien que le rituel magique exige une succession de gestes qui doivent être réalisés dans un contexte strict, tant pour l'ordre que pour le moment et l'amplitude de leur réalisation, on ne trouve que peu d'indications sur la modalité de l'énonciation magique. Il s'agit là d'un domaine qui, appartenant au seul discours oral, semble demeurer hors de portée de toute analyse textuelle et d'un problème que posent également les textes religieux. Il en va de même de l'origine et de la nature du pouvoir qui confère à la parole, dite ou écrite, la possibilité de participer à une œuvre créatrice, car s'il est dit que l'ordre de nommer les animaux provient de Dieu, rien n'explique, dans la Bible ou dans les récits mythologiques, la nature même des mots prononcés par les humains.

L'association de l'incantation au chant, autant que la pratique d'une certaine magie par des musiciens réputés, invite à analyser les descriptions de rituels incantatoires à la lueur des préceptes musicaux dont les fondements se sont établis à des époques sensiblement identiques à celles qui ont vu l'apparition et la diffusion des grimoires les plus importants. La musique, prise pour l'instant au sens large en tant qu'expression mélodique, semble posséder ou manifester un pouvoir surhumain.

Les récits mythologiques posent d'emblée le problème du rapport qui peut exister entre la parole, perçue comme une partie d'un discours linguistique, et la musique, interprétée comme une succession de sons. Par ailleurs, il reste encore à déterminer si les sons possèdent, par eux-mêmes ou seulement lorsqu'ils s'associent pour composer une mélodie, une quelconque qualité naturelle ou surhumaine. La maitrise musicale se rapporte à la connaissance des mots et des sons et pour cela il est nécessaire d'analyser, d'une part, les rituels d'énonciation dans un contexte magique et religieux et d'autre part la place que l'apprentissage et la pratique de la musique ont pu occuper dans le système cognitif médiéval.

Il est donc nécessaire, dans un premier temps, de répertorier les différents types d'incantations que l'on peut trouver dans les grimoires et de différencier les types de discours préconisés. Ces éléments pourront ensuite être analysés à l'aide des principes qui conforment le substrat de la pensée et de l'activité magique. Dans un second temps, après avoir rappelé ce que le Moyen Âge peut entendre par musique, et la place que celle-ci occupe dans la pensée occidentale, aussi bien d'un point de vue philosophique, qu'esthétique ou moral, il sera possible de déterminer le rapport que peuvent entretenir les deux catégories de mots, religieux ou magiques, avec le chant et les différentes pratiques musicales. À la lueur des caractéristiques propres au chant religieux, on pourra alors émettre une hypothèse sur les modalités d'énonciation des incantations magiques. 


\section{LE DISCOURS INCANTATOIRE}

\subsection{Les incantations magiques dans les grimoires}

Les Cyranides et le Picatrix sont sans doute les deux grimoires qui doivent être considérés comme les sources fondamentales de la pratique magique occidentale.

Les Cyranides ${ }^{8}$ se composent de quatre livres qui contiennent des prescriptions médicales et des recettes magiques; ils détaillent les rituels et les préparations à réaliser pour conjuguer et fortifier les qualités des différents éléments présentés. Les ingrédients utilisés sont présentés, par ordre alphabétique, par groupes de quatre éléments, comprenant un oiseau, un poisson, une pierre et une plante, de sorte que l'ensemble de l'œuvre tient autant du bestiaire que du lapidaire. Mélangeant l'occultisme à la science, avec des applications ou des explications parfois difficiles à déchiffrer d'un point de vue médical ${ }^{9}$, les Cyranides se caractérisent par un «syncrétisme des idées grecques et orientales qui a produit la littérature hermétique ${ }^{10}$. L'identité de l'auteur de ces livres demeure incertaine puisque les différents volumes sont traditionnellement attribués à Kyranos, roi de Perse, à Harpocration d'Alexandrie ou encore à Hermès Trismégiste. La version latine, réalisée par "un inconnu qui s'appelle modestement infimus clericus " ${ }^{11}$, date de 1168-1169. Le contenu de l'œuvre aurait ainsi évolué entre le $\mathrm{IV}^{\mathrm{e}}$ et le $\mathrm{XII}{ }^{\mathrm{e}}$ siècle. Toutefois, malgré les diverses formules magiques, qui peuvent donner l'impression d'une dispersion thématique, le sujet premier de l'œuvre, comme l'indique son titre latin complet (Liber physicalium virtutum, compassionum et curationum, collectus ex libris duobus...) ${ }^{12}$, sont les vertus et surtout les sympathies et les oppositions qui peuvent exister entre les propriétés de chaque élément ${ }^{13}$.

Le premier livre s'ouvre sur un discours hautement symbolique qui décrit une rencontre allégorique entre l'auteur de l'ouvrage et un sage vénéré. Tous les deux se dirigent vers un temple pour prier Dieu. C'est alors que l'auteur est invité à mesurer et calculer les différentes proportions du temple où il se rend ${ }^{14}$.

La présence des trois cent soixante-cinq marches pour atteindre l'autel est assez éloquente pour comprendre que les mesures indiquées sont en relation

8. Nous utilisons simultanément la version de Fernand Mély (Les lapidaires de l'Antiquité et du Moyen Âge, Tome III, Paris, 1902) et de Louis Delatte (Textes latins et vieux français relatifs aux Cyranides, Paris, 1942) qui présentent des textes très proches mais avec certaines variantes, notamment en ce qui concerne les références aux nombres.

9. David Bain, "Eight further conjectures on the Cyranides ", Scholians, 7, 1998, p. 121-125

10. Louis Delatte, Textes latins et vieux français relatifs aux Cyranides, Paris, 1942, Introduction, p. 4.

11. Ibid., p. 5.

12. Textes Latins..., p. 13.

13. Fernand Mély, "Les Cyranides ", Revue de Philologie, Paris, 1900, p. 120.

14. Fernand Mély, Les lapidaires de l'Antiquité et du Moyen Âge, Tome III, Paris, 1902, Prologue, $\$ 8$. Textes Latins..., p. 16-17. 
avec l'astronomie et que l'ensemble du temple doit être interprété comme une représentation de la Création. Cette dernière semble être régie par l'ordre et le nombre, surtout si l'on suit l'édition de Delatte qui retient les mesures de 32/78/208, toutes multiples de 4 . C'est dans ce contexte de symbolisme numérique que s'inscrivent les connaissances inscrites en syriaque, sur une colonne de métal, près de l'autel. Le sage y aurait gravé les vertus de chaque chose existante, en se limitant à 24 éléments appartenant aux quatre règnes différents de la Création (terre/plante, eau/poisson, air/oiseau, minéral/pierre) ${ }^{15}$. Autrement dit, dès le début du livre, le nombre et la proportion offrent une approche symbolique du monde matériel qui permettrait, comme le font les marches qui mènent à l'autel, de comprendre la Création et de s'élever vers Dieu. Or, le sage insiste sur le fait que les puissances divines sont innombrables et qu'il ne faut pas les énumérer. Elles échappent ainsi à la dimension terrestre, physique, de l'univers humain, en établissant un autre plan d'existence. Il ne s'agit pas d'atteindre, physiquement parlant, le plan d'existence divine, puisque le temple décrit aurait justement été construit par des géants qui voulaient atteindre le ciel. Pour ce péché d'orgueil, ils auraient été frappés par la foudre ou précipités dans les abîmes de la Crète. Pour autant le mortel peut, par la prière et les rituels exposés, dialoguer avec les puissances célestes et espérer obtenir ce qu'il désire.

On peut donc déceler dès l'introduction, un certain didactisme, une volonté d'apporter un enseignement rigoureusement exposé, bien que teinté de symbolisme et usant d'allégories. Et c'est, paradoxalement, cette rigueur qui semble manquer aux descriptions des rituels proposées par les Cyranides.

De nombreuses préparations exigent l'inscription de caractères sur un parchemin pour faire des phylactères ou encore sur des membres d'animaux pour en faire des remèdes. D'autres requièrent en plus la prononciation $\mathrm{du}$ nom du patient ou encore de différentes imprécations ${ }^{16}$, qui peuvent aller de l'énonciation d'un seul mot $^{17}$ à celle de phrases entières ${ }^{18}$. Dans ce dernier cas, la phrase explique de façon détaillée le but de la préparation. Cependant, on ne trouve aucune indication sur les modalités de lecture et d'énonciation de ces phrases ou de ces mots qui pourraient inscrire le discours incantatoire dans l'univers ordonné qui a été décrit auparavant. Faut-il lire les textes de façon monotone ou sur une hauteur de voix précise à un moment opportun?

Ces doutes sont d'autant plus contraignants que, par moments, le texte semble indiquer une prononciation de sons, plus que de paroles intelligibles. En effet, les préparations à base de vin, par exemple, sont mises sous la protection de Bacchus et c'est à lui que s'adressent tout naturellement les

15. Les Lapidaires..., Prologue, $\$ 10$; Textes Latins..., p. 17-18.

16. Les lapidaires..., II, A, $\$$ 43. Textes Latins..., p. 99-100

17. Les lapidaires..., II, E, $\$ 12$. Textes Latins..., p. 107.

18. Les lapidaires..., III, K, 8. Textes Latins..., p. 159 et IV, Y, \$2. Textes Latins..., p. 200. 
différentes énonciations magiques. Il faut donc prononcer son nom ${ }^{19}$, mais également la "formule dyonisiaque », certes christianisée par le traducteur latin mais qui se caractérise par la présence de véritables vocalises qui s'entremêlent aux imprécations ou aux invocations divines classiques ${ }^{20}$, pouvant prendre l'apparence d'une véritable formule de sacrifice avec une intonation particulière ${ }^{21}$.

Les Cyranides soulignent donc, d'une part, la différence qui peut exister entre l'énonciation de mots isolés et de phrases entières, grammaticalement explicites, et, d'autre part, la nécessité de respecter une modalité d'énonciation différente pour chaque texte. Il s'agit là d'une problématique récurrente présentée par un autre grimoire fondamental de la magie médiévale : le Picatrix ${ }^{22}$.

Le Picatrix est en réalité une traduction latine, puis castillane et française réalisée au XIII ${ }^{\mathrm{e}}$ siècle, du Ghayat al-Hakim dont l'auteur pourrait être Maslama ibn Ahmad al-Majrîtî. Ce grimoire aurait donc été composé à l'origine au $\mathrm{X}^{\mathrm{e}}$ siècle, au moment où sont introduites en Europe, via les royaumes hispaniques, de nouvelles théories astrologiques ${ }^{23}$, mystiques et ésotériques ${ }^{24}$. Cette œuvre boit aux sources grecques d'Empédocle, à Ptolémée, et s'inspire également des écrits de ben Wahsiyya, d'al-Razi et d'al-Kindi ${ }^{25}$. L'importance du Picatrix pour l'Antiquité tardive et le Moyen Âge occidental provient du fait qu'il replace l'étude et la pratique de la magie dans un contexte philosophique et moral $^{26}$. Le manuscrit, qui conseille fortement au lecteur de maîtriser d'abord les arts du quadrivium, est un exposé théorique qui adopte une représentation néoplatonicienne de l'univers ${ }^{27}$ et qui lie la pratique magique à l'étude des arts libéraux, surtout de l'astronomie et de l'arithmétique (Livre II, chapitre IV).

Divisé en quatre livres, comme les Cyranides, ce grimoire aborde les différentes facettes de la composition d'un talisman. La confection d'un objet magique dépend de la conjonction planétaire ${ }^{28}$, car chaque jour et chacun de ses moments sont placés sous une influence astrale bien précise, mais l'énonciation de mots y joue un rôle de premier ordre puisque

les mots constituent une des parties de la nigromancie, parce que les mots ont en euxmêmes une vertu nigromantique. Voilà pourquoi, dit Platon, comment un ami devient

19. Les lapidaires..., I, $\Theta, \$ 2$ et I, $\Theta, \S 6$. Textes Latins..., p. 53

20. Les lapidaires..., I, $\Theta, \mathbb{\$} 6$. Textes Latins..., p. 53-54 : «Est autem Dionysiacum nome hoc : evira sub cichi cheiv ex iii oioo acul. Veritas autem nominis est : iy evio A ; sic enim Arpocration. Kyranus vero sic : euia baicheu euileu Dionyse ».

21. Les lapidaires..., III, A, \$2. Textes Latins..., p. 142-143.

22. Picatrix, édition de Béatrice Bakhouche, Frédéric Fauquier et Brigitte Pérez-Jean, Turnhout, 2003.

23. Nicolas Weill-Parot, "Astral magic and intellectual changes " in Jan Bremmer et Jan Veenstra, The metamorphosis of magic, Leuven, 2002, p. 167.

24. Juan Vernet, Historia de la ciencia española, Barcelona, 1988, p. 59.

25. Picatrix, edición de Marcelino Villegas, Madrid, 1982, p. 13-15.

26. Picatrix, édition de Béatrice Bakhouche, Frédéric Fauquier et Brigitte Pérez-Jean, p. 6-8.

27. Ibid., p. 9

28. Ibid., p. 49. 
un ennemi par des paroles méchantes et outrageantes, à l'inverse, un ennemi devient ami par de bonnes et amicales paroles. D'où il est patent que la parole possède en soi une puissance nigromantique ${ }^{29}$.

Le Picatrix n'explique pas la nature des mots auxquels il se réfère, ni la source de leur pouvoir. Il n'explique pas, non plus, le rapport qui pourrait exister entre eux et les planètes ou les constellations. Ce sont là des éléments qu'il faudra chercher dans d'autres écrits.

Les théories magiques du Picatrix se fondent sur l'idée que l'homme est un microcosme, un reflet de la Création (Livre I, chapitre VI). Il se distingue " par son art et ses sciences $"^{30}$ et surtout par sa voix, grâce à laquelle "il imite les animaux à sa guise (...) produit tous les autres sons de tous les êtres vivants, et change leur formes et leur apparences à sa guise $"^{31}$. Le pouvoir de la voix, qui ne se limite pas à la seule émission de la parole, est alors développé dans le troisième livre où trois chapitres consécutifs indiquent comment s'adresser aux esprits des différentes planètes.

Le mage doit d'abord se purifier, en lavant son corps et en revêtant des habits propres aux couleurs de l'astre auquel il s'adresse, pour ensuite réciter "brièvement les demandes adaptées à chaque planète "32, ce qui laisse supposer que le discours adressé aux esprits planétaires ne doit pas être complexe, linguistiquement parlant. Pourtant, le Picatrix rapporte plusieurs prières qui comptent plus d'une dizaine de lignes. Ce qui est important, néanmoins, c'est que le grimoire indique toujours, à la fin du discours, la nécessité d'introduire la motivation spécifique de la prière par des formules telles que « je te demande de faire pour moi telle ou telle chose ", « je te conjure de faire pour moi telle ou telle chose ".

Dans la description de chaque rituel, l'auteur insiste sur l'attitude que le mage doit adopter pour s'adresser aux forces surhumaines, en agissant avec " humilité, tristesse et douceur " ${ }^{33}$, " avec courage et sans crainte " ${ }^{34}$, ou encore "à la manière des ermites et des chrétiens ${ }^{35}$. On peut alors supposer que le ton de la voix doit refléter cet état d'esprit. De même, la demande peut être répétée " une fois " ou "souvent ", ce qui implique une reproduction à l'identique et donc un timbre de voix et une cadence mesurés et pré-établis, de sorte que ces textes apparaissent comme des formules, qui seraient normalisées pour tous les mages. L'identification de ces discours magiques comme des prières impose de déterminer ce qui peuvent les différencier des prières traditionnellement associées à une pratique religieuse. Nous aborderons dans un travail ultérieur l'analyse structurelle, purement sémiologique, de ces discours aussi bien religieux

\footnotetext{
29. Ibid.

30. Ibid., p. 80.

31. Ibid., p. 80-81.

32. Ibid., p. 209.

33. Ibid., p. 217.

34. Ibid., p. 224.

35. Ibid., p. 217.
} 
que magiques. Mais il est déjà possible de remarquer que le Picatrix n'explique pas vraiment comment énoncer ces formules, seule la comparaison de ces discours magiques avec les prières et les chants religieux pourrait apporter un début de réponse à cet aspect du rituel incantatoire. Dans le cadre de l'analyse musicale, il est alors nécessaire de déterminer les aspects techniques, moraux et spirituels qui peuvent différencier l'enchantement magique du chant religieux.

En outre, aux longues prières s'ajoutent également les noms des différents esprits que le mage veut voir agir en sa faveur. À plusieurs reprises, le Picatrix rappelle que les noms utilisés au Moyen Âge, ne sont que des variantes de noms plus anciens, " ceux qu'utilisaient d'ordinaire ceux qui priaient les planètes et ils avaient l'habitude de les utiliser dans les prières qu'ils adressaient aux planètes " ${ }^{36}$. Mars, par exemple, est appelé "Mara smyt, nom qui signifie maître des malfaiteurs ${ }^{37}$. Mais il existe aussi des noms plus génériques, qui regroupent les différents noms attribués aux différentes parties ou qualités de chaque planète ${ }^{38}$. En s'adressant à l'une ou à l'autre, le mage doit employer un nom bien précis. C'est ainsi qu'il doit énumérer de une à sept fois diverses listes de noms ${ }^{39}$ qui représentent les qualités de l'esprit planétaire que le mage veut faire agir. Il ne s'agit plus d'un discours structuré, comme les présentent les Cyranides ou les prières mentionnées plus haut par le Picatrix lui-même. Ce sont des noms, des mots isolés, qui doivent exprimer par eux-mêmes l'état d'esprit et la volonté du mage, sans que l'auteur du texte donne de plus amples indications.

D’autres grimoires, inspirés par ces deux premiers textes, reprennent les mêmes dispositions à quelques détails près pour l'énonciation des prières et des invocations spirituelles.

Le dénommé Libro de Astromagia, composé au XIII ${ }^{\mathrm{e}}$ siècle ${ }^{40}$ sous la direction d'Alphonse X de Castille, correspond à un volume inachevé et anépigraphe qui ne peut en aucune façon être confondu avec le Picatrix.

Bien qu'actuellement le manuscrit ne présente que quatre livres, il devait, à l'origine du projet, en compter sept et constituer une grande compilation de thèmes magiques et planétaires ${ }^{41}$, synthétisant au mieux la théorie et la pratique des doctrines astromagiques ${ }^{42}$. Comme les autres traités, il propose des recettes magiques d'ordre médicinal, pour altérer la volonté des hommes ou la réalité du monde, mais en utilisant presque exclusivement des talismans. Les prières adressées aux esprits planétaires pour parfaire le rituel occupent parfois un folio entier. Elles apportent quelques indications sur l'attitude du mage,

36. Ibid., p. 248-249.

37. Ibid., p. 240.

38. Ibid., p. 248.

39. Ibid., p. 250.

40. Tratado de astrología y magia de Alfonso Xel Sabio, ed. de Carlos Alvar, Alfonso D'Agostino y Demetrio Santos, Valencia, 2000, p. 45.

41. Ibid.

42. Alejandro García Avilés, "Imágenes mágicas. La obra astromágica de Alfonso X y su difusión en la Europa bajomedieval » in Miguel Rodríguez Llopis, Alfonso X, aportaciones de un rey castellano a la construcción de Europa, Murcia, 1997, p. 140. 
mentionnant ses larmes et ses pleurs ${ }^{43}$, ou sur sa déclamation ${ }^{44}$, qui doit être puissante comme une clameur. Ce sont toujours des structures fixes qui finissent par une variante qui permet d'introduire la demande effectuée ${ }^{45}$. Ces demandes sont parfois accompagnées de gestes et de révérences ${ }^{46}$, surtout lorsqu'il s'agit de répéter les noms des esprits avant et après l'opération magique ${ }^{47}$. On peut supposer que les révérences répétées s'accordent avec l'énonciation des noms qui semblent, par ailleurs, impliquer une certaine cadence, contrairement aux longues périodes d'incantation. Mais encore une fois rien n'est précisé sur la tonalité musicale qui doit être appliquée aux noms prononcés.

Le dernier grimoire que nous mentionnerons à présent est le De Nigromancia, dont l'importance tient d'avantage à son attribution à Roger Bacon, et à la référence à certaines valeurs numériques, qu’à son contenu, fort peu original.

Ce manuscrit qui pourrait être un apocryphe composé au XVI ${ }^{\mathrm{e}}$ siècle, insiste sur la nécessité de répéter les prières 7 fois dans chaque direction ${ }^{48}$, ce qui donne au discours énoncé une dimension cosmique. En effet, si les quatre directions peuvent se rapporter à la dimension terrestre, les sept répétitions ne font que rappeler les sept planètes et donc établir ainsi un lien entre la terre et le ciel. On peut alors se demander quelle influence peuvent avoir les conceptions astronomiques dans l'énonciation d'un discours rituel, magique ou religieux. Ce rapport ne fait d'ailleurs que s'inscrire dans les limites des conseils prônés par le Picatrix de prier en accord avec la personnalité de chaque planète pour obtenir un effet particulier. Le De Nigromancia impose également d'adopter une attitude particulière au moment de l'incantation, même si elle ne correspond pas toujours à l'attitude humble préconisée par les autres grimoires. Il est certes nécessaire de prier avec une grande dévotion et solennitét ${ }^{4}$, mais il faut également s'agenouiller avec dignitée ${ }^{50}$. Ce sont là des attitudes, des sentiments qui doivent être en accord, et exprimés par le ton et la fermeté de la voix qui accompagne le rituel.

On l'aura compris, les descriptions des différents rituels magiques visent à guider l'attitude du mage et à figer les étapes de l'action magique. Cependant, on se heurte à la modalité de formulation des prières magiques qui devrait également répondre à la vision ordonnée et structurée de la Création que propose la pensée magique, mais pour laquelle il n'y a pas de véritable précision. L'incantation peut prendre la forme de longues périodes ou de mots isolés répétés et, dans une certaine mesure, elle semble influencée, voire dictée, par la personnalité de l'esprit planétaire auquel elle s'adresse.

43. Tratado de astrología, fol. $30 \mathrm{r}^{\circ}$.

44. Ibid., fol $31 \mathrm{r}^{\circ}$.

45. Ibid., fol. $30 \mathrm{r}^{\circ}$.

46. Ibid., fol. $32 \mathrm{r}^{\circ}$.

47. Ibid., fol. $33 \mathrm{v}^{\circ}$.

48. Roger Bacon, De Nigromancia, édition de Michael Albion Macdonald, New Jersey, 1988, p. 10.

49. Ibid., p. 31.

50. Ibid., p. 43. 
En outre, les différentes prescriptions magiques ainsi présentées posent le problème du savoir et du savoir-faire. Les Cyranides exposent d'abord une étude des qualités et des propriétés de chaque élément appartenant aux différents règnes de la Création, et le Picatrix insiste sur le besoin de bien dominer les fondements de l'astronomie pour agir en accord avec les puissances planétaires. Pour autant, toutes les recettes semblent couler de source, il suffit de suivre pas à pas les étapes décrites, de les réaliser au bon moment, pour que l'incantation réussisse. Tout se passe comme si, en réalité, il n'était pas nécessaire de connaître l'origine ou la signification des mots utilisés. D'ailleurs, le Picatrix explique, très rapidement, l'origine des mots et des noms utilisés lors des rituels, alors que les Cyranides et le Libro de Astromagia sont beaucoup plus succincts sur ce sujet. De ce fait, on peut se demander si l'enchanteur sait toujours ce qu'il dit, s'il a conscience des fondements théoriques qui constituent le substrat de l'incantation magique. C'est le problème essentiel posé par les interdictions du Concile de Braga II. Les incantations condamnées par les canons conciliaires sont liées à des activités du peuple (tisser, cueillir des plantes), réalisées par des individus qui, il est légitime de le penser, ne maîtrisaient pas les arts libéraux et ne connaissent pas les théories astronomiques ou musicales de leur époque. Il semble donc exister deux types d'enchanteurs, le premier agit par mimétisme, le second étudie les sciences du quadrivium et de la Nature. Ce dernier serait alors le plus susceptible d'apporter des explications valables sur la source du pouvoir des mots.

\subsection{Théories magiques sur le mot}

Sans entrer ici dans la polémique de savoir à quel point Roger Bacon était convaincu des théories magiques qu'il défendait dans son Opus Maius, on ne peut que souligner l'initiative qu'il prend de nuancer ses arguments dans ses Prodiges de la Nature et de l'Art ${ }^{51}$. Dans ce document adressé au pape Nicolas III, le franciscain veut se défendre contre les accusations de pratiquer la magie portées à son encontre. Il tente de démontrer que cette pratique est très inférieure aux lois divines et à la capacité de l'homme d'agir, en utilisant sa raison et son savoir. En ce qui concerne le pouvoir des mots magiques, et la nécessité de les prononcer en respectant une hypothétique intonation particulière, Roger Bacon commence le second chapitre avec une position tranchée, car pour lui « quant à ce qu'il faut penser des formules et des caractères magiques, voici : il est hors de doute que ces choses sont fausses ou douteuses, ou même irrationnelles ${ }^{52}$. Il affirme ainsi que

les magiciens mettent stupidement leur confiance dans les incantations et les caractères parce qu'ils leur supposent de la puissance ; ils ne s'occupent que de cela, délaissent les

51. Roger Bacon, Lettre sur les prodiges de la nature et de l'art, édition de Albert Poisson, Paris, 1977.

52. Ibid., p. 15. 
œuvres de la nature et de l'art, s'adonnent aux erreurs de leurs talismans et évocations. C'est ainsi que, poussés par leur stupidité, tous ces hommes sont privés de la véritable science $^{53}$.

Roger Bacon ne nie pas le pouvoir des paroles, il établit simplement que certains individus leur attribuent des qualités qu'elles n'ont pas. Ceux qui s'adonnent à l'étude de la Nature comprennent mieux ce qu'est le pouvoir des mots. Le franciscain nuance sa position en établissant que " tout agent manifeste sa force et sa nature sur une matière extérieure (...) C'est pourquoi l'homme peut projeter sa puissance et sa force hors de lui d'autant qu'il est le plus noble de la création ${ }^{54}$. Il en conclut que, alliant l'imagination au désir de l'âme, "l'homme par paroles ou par actions, peut réellement produire des prodiges " $"$. Le religieux explique cette faculté des paroles en affirmant qu'elles "naissent à l'intérieur, formulées par les pensées de l'âme et le désir " ${ }^{56}$ et qu' elles se manifestent à l'extérieur en passant par les "voies ouvertes sur le cœur et les organes intérieurs ${ }^{57}$, de sorte qu'elles recueillent la santé, la maladie, les émotions de l'émetteur qui peuvent, à leur tour, affecter l'environnement de l'individu. La conclusion ne se fait donc pas attendre. Roger Bacon accepte que la simple émission de la parole puisse produire des phénomènes naturels, pour peu que l'émetteur agisse sous l'emprise d'un désir violent ou manifeste une ferme intention d'obtenir ce qu'il désire. Il ne s'agit pas pour lui d'attribuer aux sons un quelconque pouvoir surhumain mais tout simplement de reconnaître que « la voix vive a une grande vertu, non pas qu'elle ait la puissance que lui attribuent les magiciens pour corrompre, comme ils le prétendent, mais elle peut opérer selon les lois naturelles " ${ }^{58}$, en l'occurrence celle qui établit comment tout agent a la capacité de manifester sa force sur une matière extérieure. Ce point de vue, pour le Moyen Âge, dans le domaine de la magie et dans l'analyse des fondements scientifiques de la parole, rappelle les préceptes établis par l'autre grand traité de théorie magique, le De Radiis de al-Kindi, qui aurait fortement influencé la pensée de Bacon ${ }^{59}$.

Dans une interprétation platonicienne de la Nature et de la Création, al-Kindi perçoit le ciel divisé en sphères régies par leur planète respective. Pour lui,

chaque étoile a, en effet, sa nature propre et ses manières d'être dans lesquelles est contenue, entre autres, la projection de rayons. Et de la même manière que chacune a sa nature propre, qui ne peut se retrouver totalement en aucune autre et dans laquelle est contenue l'émission de rayons, de même ces rayons sont de nature différente dans les diverses étoiles, comme les étoiles elles-mêmes sont différentes selon leur nature ${ }^{60}$.

53. Ibid., p. 16.

54. Ibid., p. 21.

55. Ibid., p. 25.

56. Ibid., p. 25.

57. Ibid., p. 26.

58. Ibid., p. 26.

59. Nicolas Weill-Parot, Les images astrologiques au Moyen Âge et à la Renaissance, Paris, 2002, p. 331-333.

60. Al-Kindi, De Radiis, édition de Didier Ottaviani, Paris, 2003, p. 13. 
La pensée kindienne se construit sur l'idée que

l'état de l'harmonie céleste est tel que, comme toutes les étoiles ont des natures diverses et que, de ce fait, tous leurs rayons des effets différents, il arrive que ces effets s'aident mutuellement dans le monde des éléments vis-à-vis d'une même matière ou s'entravent pour une autre ${ }^{61}$,

établissant ainsi une idée harmonique, c'est-à-dire complémentaire et interactive, du monde. Bien entendu, cette même idée d'harmonie cosmique renvoie directement aux théories de la musique des sphères et de leur influence dans le domaine de la production musicale que nous aborderons par la suite. Mais, pour l'instant, retenons que al-Kindi établit l'existence d'une personnalité propre à chaque astre qui se manifeste également dans les rayons que chaque corps céleste produit et qui inondent l'ensemble de la Création. Or, tout comme les étoiles émettent des rayons, les sons eux-mêmes, "produits en acte, émettent des rayons comme le font les autres choses en acte " ${ }^{62}$. Le rapport entre les sons et l'émission des rayons établit une relation supplémentaire entre les sons et les planètes, les premiers étant individuellement reliés aux secondes ou à une configuration astrale spécifique ${ }^{63}$, participant ainsi à l'idée d'interrelation harmonieuse entre les différents composants de l'univers.

Le chapitre VI du De Radiis est alors spécifiquement dédié à l'usage des mots en y passant « insensiblement de la parole normale à la parole magique, (...) parce que l'une et l'autre procèdent d'une même explication " ${ }^{64}$ issue de l'idée d'harmonie universelle qui « donne leur effet aux sons, ainsi qu'aux herbes et à d'autres choses et, de la même manière, elle donne des effets qualitativement très variés dans des choses différentes ${ }^{65}$.

Or, en parlant de sons, al-Kindi ne se réfère pas uniquement aux manifestations sonores naturelles, mais également aux mots prononcés par l'homme et dont le sens premier peut ne pas être connu de celui qui les utilise ${ }^{66}$. Dûment prononcés, ces mots transforment la création, ils peuvent même altérer la volonté des hommes et des animaux ${ }^{67}$. L'idée de prononciation rituelle est capitale d'autant plus que, d'après le philosophe,

parmi de tels mots, certains signifient quelque chose pour les hommes du lieu où ils sont prononcés, [ils] ne signifient rien pour d'autres, en fonction de l'attribution des hommes. Mais si les mots qui n'ont pas de signification pour celui qui les prononce obtiennent l'effet qu'il escomptait, ils le tirent de leur nature qui leur a été donnée par l'harmonie, de même qu'elle le fait en introduisant un pouvoir dans les plantes ${ }^{68}$.

61. Ibid., p. 20.

62. Ibid., p. 41.

63. Ibid.

64. Nicolas Weill-Parot, op. cit., p. 158.

65. De Radiis, p. 41.

66. Nicolas Weill-Parot, op. cit., p. 158.

67. De Radiis, p. 53.

68. Ibid., p. 49. 
La théorie kindienne ne défend pas une compréhension sémiologique pure des mots prononcés, elle accepte cependant la diversité des sons, comme la diversité de la Création, ainsi que la diversité de leur apparence et de leurs conséquences, tout en établissant que la source de cette diversité est unique et se trouve dans l'harmonie céleste ${ }^{69}$.

La capacité de l'homme à utiliser et à transmettre cette même harmonie provient du fait que,

par son être correctement proportionné, [l'homme] se présente comme semblable au monde lui-même. C'est pourquoi nous disons qu'il est un petit monde, et qu'il reçoit, comme le monde, le pouvoir d'induire un mouvement dans une matière appropriée grâce à son action, à condition d'avoir d'abord élaboré dans son âme une imagination, une intention et une certitude ${ }^{70}$.

L'action évoquée par le philosophe tient autant à la proportion correcte, à l'harmonie, du corps humain qu'à la faculté d'émettre des sons, soumise à l'imagination et à la raison humaines, tant et si bien que " pour que l'effet se produise, il faut toujours qu'il y ait, chez celui qui profere les sons, l'intention et l'imagination de la forme qu'il désire voir venir s'actualiser dans la matière grâce à la prononciation des sons $»^{71}$. Al-Kindi laisse ainsi entendre que le pouvoir du son, du mot ou de l'incantation ne provient pas spécifiquement de l'intelligibilité grammaticale du discours émis, établissant ainsi une distinction entre une linguistique magique et une linguistique matérielle ${ }^{72}$. Certes, le philosophe arabe reconnaît que l'association, raisonnée ou non, des mots peut fortifier et nuancer leurs effets ${ }^{73}$, tout comme les différents rayons peuvent se compléter pour permettre la manifestation de la réalité matérielle. Mais, en affirmant que " tous les mots prononcés produisent un certain changement dans l'ensemble de la matière élémentaire, mais celui-ci est plus grand si celui qui les prononce y ajoute une intention, et plus grand encore si se produit l'action requise par la nature en vue de cet effet $"{ }^{74}$, al-Kindi n'écarte pas la dimension linguistique du discours magique, tout en lui associant un nouvel agent qui correspondrait à la volonté, aux passions, de l'individu qui émet ce discours. Ces passions traduisent alors un état d'âme et, surtout, une qualité du discours " indicatif ou impératif, optatif, de déprécation, de supplication ou de malédiction $»^{75}$ qui requièrent une attitude et une intonation propres. Le discours impératif, par sa forme et son énonciation, peut difficilement se confondre avec, par exemple, un discours de supplication. Néanmoins, tous requièrent d'être " conjoints avec autre chose pour que l'effet se produise " ${ }^{76}$.

\author{
69. Ibid., p. 43. \\ 70. Ibid., p. 35. \\ 71. Ibid., p. 43. \\ 72. Nicolas Weill-Parot, op. cit., p. 160. \\ 73. De Radiis, p. 49. \\ 74. Ibid., p. 51. \\ 75. Ibid., p. 50. \\ 76. Ibid., p. 40.
}


Cette autre chose peut être l'ensemble de la gestuelle que le philosophe lui-même aborde (Chapitre V, $\$ 14$ ) mais, dans le contexte de l'énonciation des sons et des mots, al-Kindi souligne que " quelle que soit la certitude du suppliant, l'effet s'ensuit, pourvu qu'il prononce la supplication avec un désir souverain et avec la solennité requise pour l'action ou un autre mouvement ${ }^{77}$.

Cette solennité semble être aussi importante que l'idée de proportion ou d'harmonie et que le sens des mots par eux-mêmes, car

les mots prononcés avec la solennité requise ont cependant l'effet et la propriété de transformer tous les éléments en certaines formes nouvelles, ainsi que d'en engourdir leurs formes naturelles afin qu'ils ne produisent pas ce qu'ils auraient effectué dans le cours ordinaire des choses ${ }^{78}$.

Les mots et les sons émis par l'homme tirent leur pouvoir de l'harmonie universelle. Ils participent de la création parce qu'ils possèdent un rayonnement similaire à celui des étoiles et parce que l'homme qui les émet possède une harmonie propre qui fait de lui un reflet du macrocosme. Les mots peuvent augmenter leur pouvoir s'ils se combinent entre eux pour composer un discours clair et compréhensible, mais à l'origine ils possèdent un pouvoir propre lié aux rapports qui peuvent exister entre certains sons et les planètes. Cette diversité se traduit par des incantations magiques de natures différentes qui manifestent un état d'esprit particulier, adapté à la situation du mage et à l'esprit planétaire auquel il s'adresse. Cette adaptation implique un comportement, un état d'esprit et une expression de la volonté du mage adaptés aux contextes dans lesquels se réalisent les incantations. L'idée de solennité est alors reprise dans plusieurs grimoires comme étant indispensable au succès du rituel, puisqu'elle peut manifester la conviction et l'intensité de l'imagination du mage. Il s'agit en réalité d'une attitude mesurée qui permet d'entrer en communion, de canaliser et d'utiliser l'harmonie céleste.

La nature des sons, leur rapport avec les planètes et le microcosme humain sont des aspects dont il faut tenir compte pour tenter de déterminer les règles de modulation d'un discours incantatoire. Or, ce dernier est également influencé par les notions de mesure, d'harmonie, de proportion et de solennité qui inscrivent l'incantation magique dans un registre musical. C'est donc dans les traités de musique que nous pouvons trouver les éléments manquants dans les descriptions des rituels magiques pour mettre au jour les requis d'une telle énonciation. 


\section{LA THÉORIE MUSICALE DES ORIGINES MYTHIQUES AUX ARTS LIBÉRAUX}

\subsection{Textes sources et mythes fondateurs}

La nature de la musique et la place qu' elle occupe dans la culture médiévale ont fait l'objet de divers traités ou encore de chapitres encyclopédiques spécifiques, de longueur variable, écrits par différents auteurs qui se sont souvent influencés mutuellement.

Le Didascalion d'Hugues de Saint-Victor ${ }^{79}$ ne lui dédie qu'un court passage, en reprenant la définition isidorienne et en rappelant la triple nature de la musique selon Boèce. Des auteurs plus tardifs, comme Thomas de Cantimpré ou Brunetto Latini présentent rapidement une approche morale et spirituelle de la musique ou encore ses origines mythiques avec le personnage de Joubal ${ }^{80}$. D'après Jacques Verger, entre le XII ${ }^{e}$ et le XIII ${ }^{e}$ siècles, les auteurs les plus prolifiques en la matière auraient été Vincent de Beauvais et Barthélemy l'Anglais ${ }^{81}$. Le dominicain met " en avant la structure mathématique de la musique et les rapports rigoureux qui, de ce point de vue, l'unissent à la fois au macrocosme et au microcosme ${ }^{82}$, tandis que le franciscain veut " présenter les cadres théoriques de la science musicale et légitimer son appartenance au domaine des disciplines libérales ${ }^{83}$.

Ce sont là des domaines que nous aborderons plus spécifiquement, mais qui permettent de souligner pour l'instant que, malgré la profusion, plus ou moins réelle, des écrits sapientiels, ces derniers ne véhiculent pas d'idées originales, pour la simple raison que les encyclopédies médiévales sont essentiellement des compilations ${ }^{84}$. Or, les deux sources les plus importantes pour Vincent de Beauvais et Barthélemy l'Anglais demeurent Boèce et Isidore de Séville ${ }^{85}$. Cet état de fait semble être valable pour des écrits de la fin du Moyen Âge, comme le Tratado de Musica ${ }^{86}$, d'auteur anonyme, conservé à la Bibliothèque de l'Escurial. Ce manuscrit du $\mathrm{XV}^{\mathrm{e}}$ siècle rappelle que la musique, profane et religieuse, a été ordonnée par divers noms illustres, d'Orphée à saint Grégoire, et son manuscrit présente des passages écrits en castillan et en latin inspirés, traduits ou recopiés d'auteurs classiques, principalement saint Augustin, Boèce et Isidore de Séville ${ }^{87}$. L'intérêt de cette œuvre réside surtout dans le fait

79. Hugues de Saint-Victor, Didascalion, édition de M. Lemoine, Paris, 1991, p. 101-105.

80. Jacques Verger, «La musique et le son chez Vincent de Beauvais et les encyclopédistes du $\mathrm{XIII}^{\mathrm{e}}$ siècle »in Olivier Cullin, La place de la musique dans la culture médiévale, Turnhout, 2007, p. 72.

81.Ibid.

82. Ibid., p. 78.

83. Ibid., p. 84.

84. Ibid., p. 73.

85. Ibid., p. 76 et 83.

86. Tratado de Musica, ms. C-III-23, Biblioteca de El Escorial.

87. Ibid., fol. $1 \mathrm{r}^{\circ}-2 \mathrm{v}^{\circ}$. 
qu'elle démontre la permanence de certains éléments fondamentaux (origines mythiques et rapport avec le nombre) de ce que nous appellerons la théorie musicale.

C'est donc principalement aux œuvres de Boèce et d'Isidore de Séville que nous nous reporterons pour comprendre ce que pouvait être la musique et sur quelle conception pouvait se construire l'idée du chant, et de l'enchantement, au Moyen Âge. Pourtant, bien que le De institutione musica de Boèce demeure « tout au long du Moyen Âge la référence majeure et le creuset conceptuel et spéculatif pour la construction du discours sur la musique et, in fine, pour la rationalisation des pratiques musicales, du chant liturgique et des pratiques polyphoniques " ${ }^{88}$ et que les Étymologies isidoriennes apparaissent comme une source essentielle pour l'étude musicale, on ne peut nier à d'autres auteurs, comme Albert le Grand ou saint Augustin, qui aurait inspiré l'œuvre cassiodorienne ${ }^{89}$, d'avoir contribué à la construction de ce que pouvait être la science musicale médiévale, aussi bien du point de vue théorique que moral, philosophique, astronomique ou magique.

Un des premiers éléments qui peut différencier les auteurs, et qui influence fortement leur approche personnelle de la théorie musicale, est sans doute l'histoire de la naissance de la musique.

Sur ce point, le Tratado de Musica rappelle les différentes origines supposées de la pratique musicale, en insistant sur les noms de Joubal et de Pythagore ${ }^{90}$ qui, avec Orphée, forment la triade fondatrice de la musique médiévale et par laquelle on comprend que le Moyen Âge a " tissé des liens profonds et continus avec une Antiquité gréco-latine dont les conceptions musicales spécifiques forment son horizon convenu ${ }^{91}$.

Le personnage de Joubal est présenté dans le livre de la Genèse comme le "père de tous ceux qui jouent de la cithare et du chalumeau ${ }^{92}$. La Bible, laisse donc entendre que la pratique musicale, qui a la faculté de transformer l'auditoire ${ }^{93}$, est principalement d'ordre instrumental. La triade des instruments (à cordes, percussion et vent) minimise la présence de la vocalise qui semble se manifester plus spécifiquement dans des situations d'un intensité émotionnelle extrême, comme peuvent l'être la guerre ${ }^{94}$ ou la louange divine $e^{95}$. Or, la production vocale est toujours accompagnée par un instrument qui, au demeurant, devient l'image de la voix divine elle-même ${ }^{96}$, établissant ainsi une hiérarchie entre

88. Boèce, Traité de la musique, édition de Christian Meyer, Turnhout, 2004, Introduction, p. 15.

89. Olivier Cullin, Brève histoire de la musique au Moyen Âge, Paris, 2002, p. 33.

90. Tratado de Musica, fol. $1 \mathrm{r}^{\circ}$.

91. Olivier Cullin, Brève histoire..., p. 17.

92. Gen 4, 21

93. 2 Rs, 3, 15; I Sam., 10, 5-6.

94. Jos. 6, 4-20

95. 1 Livre des Chroniques, 15, 16-24 ; Ap. 5, 8.

96. Ap, 14, 2. 
l'instrumentiste, la production mélodique et les puissances célestes ${ }^{97}$. Cette hiérarchie soulève le problème de l'adéquation de chaque type de mélodie, de ce qu'on pourrait identifier comme un discours musical, aux différentes interventions divines souhaitées.

On l'aura compris, nous retrouvons ici les mêmes problématiques que nous soulevions en abordant la pratique et la compréhension des incantations magiques.

Pour autant, l'approche biblique n'explique pas à elle seule la genèse du discours musical.

Boèce associe la musique au personnage de Pythagore et au cheminenement déductif $^{98}$ que ce dernier a suivi pour établir les fondements de l'art musical. L'approche philosophique et mathématique soulève le problème du rapport entre les sens et la raison, mais également de la perception du monde et de l'harmonie des choses par l'idée de proportion ${ }^{99}$. Pythagore, selon Boèce, n'invente pas la musique, il ne fait qu'expliquer les consonances, c'est-à-dire les fusions des sons. Ces derniers sont perçus comme ayant des propriétés intrinsèques, de sorte qu'ils puiseraient leurs propriétés et leurs natures ailleurs que dans l'industrie humaine. Ces propriétés demeurent liées à des proportions arithmétiques, si bien que «Boèce fait de la musique une science mathématique en relation avec Pythagore ${ }^{100}$. Cette transformation implique, d'un point de vue chronologique, que la musique et la fabrication d'instruments étaient antérieures à la compréhension des lois qui régissent les proportions musicales. Le savoir-faire aurait ainsi précédé le savoir, mais il n'en demeure pas moins vrai que le cheminement intellectuel exposé par Boèce pose le problème de différencier l'émission de sons aléatoires de la production musicale elle-même.

Plus tard Isidore de Séville conjugue l'histoire de Joubal à celle de Pythagore ${ }^{101}$, en y adjoignant également d'autres noms (Linus de Thèbes, Zéthos, Amphion). La référence isidorienne au philosophe établit le rapport de la musique aux mathématiques et à la symbolique des nombres ${ }^{102}$. La musique est ainsi inscrite dansl'étude de l'arithmétique, aussi bien d'ordre calculatoire que d'interprétation symbolique des nombres ${ }^{103}$. Elle confirme donc son appartenance aux sept arts libéraux qui, dans une approche augustinienne, "doivent servir de degrés assurés pour aller des choses corporelles aux incorporelles » ${ }^{104}$, ce qui lui permet

97. Pour une étude de la hiérarchie des instruments et de leurs origines mythiques voir Jean Marie Fritz, "La réception médiévale des mythes antiques d'invention de la musique », in Olivier Cullin, La place de la musique..., p. 23-38.

98. Umberto Eco, Art et beauté dans l'esthétique médiévale, Paris, 1987, p. 57 et 59.

99. Ibid., p. 45-51.

100. Ibid., p. 58.

101. Isidore de Séville, Etimologías, édition de José Oroz Reta et Manuela Marcos Casquero, 2 vol., Madrid, 2000, III, 16, 1.

102. Porfirio, Vida de Pitágoras, ed. de Miguel Periago Lorente, Madrid, Cátedra, 1987, Introducción, p. 15.

103. Etimologias, note 31, p. 444.

104. Saint Augustin, De musica, édition de Messieurs Thénard et Citoleux, Paris Sandre, 2006, réédition de l'édition de 1864, et préfacée par Anne-Isabelle Thénard-Touboulic, p. 5. 
d'établir un lien avec les incantations magiques, elles aussi identifiées comme des discours adressés à l'au-delà.

La concordance, la qualité discursive de la musique, son implication dans la manifestation du divin et dans la compréhension de la Création sont autant de paramètres qui orientent les théories musicales qui se sont développées pendant tout le Moyen Âge et qui ne pouvaient pas manquer d'influencer la pratique des incantations. Leur analyse doit permettre de comprendre quelles pouvaient être les modalités d'énonciation des incantations, ainsi que les critères spécifiques qui pouvaient différencier les prières magiques des prières religieuses.

\subsection{Saint Augustin et la bonne modulation}

Parmi les différents traités qui portent sur la musique, le De musica ${ }^{105}$ de saint Augustin occupe une place prépondérante à plusieurs titres. Tout d'abord, la comparaison des écrits de Boèce et de saint Augustin ${ }^{106}$, permet de remarquer que le premier auteur « ne dit rien de la rythmique ou de la métrique, matières pourtant traditionnelles de l'enseignement de la musique dans l'Antiquité. Les lecteurs médiévaux recueilleront cet enseignement dans le De Musica de saint Augustin " ${ }^{107}$. Les travaux de ces deux auteurs sont donc complémentaires en ce qui concerne le développement théorique, purement sapientiel, des lois musicales, mais, pour ce qui est de la teneur spirituelle, l'œuvre de saint Augustin revêt une valeur toute particulière. Augustin aurait écrit son traité vers 386 période qui correspond à son baptême, si bien que ses réflexions sont imprégnées de son cheminement spirituel et intellectuel. D’ailleurs, Augustin se réfère à la musique dans d'autres écrits comme dans les Rétractations (386) ou encore les Confessions (397-401) où il expose des points de vue parfois contradictoires sur le sujet, témoignant ainsi de l'évolution personnelle qu'il a lui-même connue.

L'évêque d'Hippone établit une définition en apparence simple, selon laquelle "la musique est une science qui apprend à bien moduler " ${ }^{108}$, ou encore "la science des mouvements bien ordonnés " ${ }^{109}$, en spécifiant que la bonne modulation se rapporte à " l'art dans les mouvements, ou du moins l'art d'exécuter des mouvements réguliers ${ }^{110}$. La régularité des mouvements renvoie à la notion de cadence, "à la mesure naturelle des paroles et des

105. Sauf indication autre nous utiliserons l'édition numérique des Euvres complètes de saint Augustin, Traduites pour la première fois en français sous la direction de M. Poujoulat et M. l'abbée Raulx, 1864-1872, réalisée par l'Abbaye Saint-Benoît de Port-Valais, 1998.

106. Pour une correspondance des sources et des thèmes entre sain Augustin et Boèce, voir Ubaldo Pizzani, «Du rapport entre le De Musica de S. Augustin et le De Institutione Musica de Boèce » in Alain Galonnier, Boèce ou la chaîne des savoirs, Louvain-Paris, 2003, p. 357-377.

107. Christian Meyer, op. cit., p. 14.

108. De musica, I, 2.

109. Ibid., I, 3.

110. Ibid., I, 2. 
sons " ${ }^{111}$ faisant ainsi de la musique la science " qui a rapport avec ce qu'on nomme le rythme ${ }^{112}$. D'un point de vue technique, la musique augustinienne " ne concerne pas ce que nous appelons actuellement musique (mélodie et harmonie) mais le rythme de la prosodie ${ }^{113}$, ce qui peut expliquer pourquoi la réalisation instrumentale est totalement absente dans le De musica ${ }^{114}$.

C'est l'énonciation modulée et raisonnée des mots, en accord avec un rythme prédéfini, qui compose le fondement et la manifestation sublime de la musique augustinienne. Elle peut préfigurer en cela la théorie cassiodorienne selon laquelle " la parole est musique en elle-même ${ }^{115}$. Le De musica réserve d'ailleurs plusieurs chapitres (Livres II à V) à l'identification des différents vers, des pieds qui les composent et des accents qui doivent ponctuer leur énonciation. Il ne s'agit pas d'émettre des sons isolés, mais de construire une phrase musicale, composée de divers sons enchaînés ou entrecoupés par des silences qui vont rythmer la phraséologie du discours ${ }^{116}$.

Or, Augustin ajoute un élément supplémentaire qui dépasse celui de la seule réalisation technique, car "le même mouvement ne paraît pas bien, quand il manque d'à-propos, encore qu'il semble conforme aux lois de la cadence $"{ }^{117}$. Il allie au savoir-faire de la technique le bon jugement de l'exécutant, qui adapte son action au contexte. La bonne modulation n'est pas seulement une affaire de décompte syllabique, mais aussi de raison. C'est cette particularité qui permet de faire la distinction entre l'histrion, qui imite un savoir-faire sans vraiment comprendre les fondements de l'art qu'il pratique ${ }^{118}$, du musicien qui pratique une science ${ }^{119}$. Cette dernière, considérée comme le "privilège de l'âme " ${ }^{120}$, place la théorie et la pratique musicales sur un plan supérieur à celui de la production mélodique.

La musique augustinienne semble d'ailleurs mieux identifiée par ce qu'elle n'est pas que par ce qu'elle est. Alors que la connaissance musicale est ignorée de l'histrion, elle est également inconnue du grammairien, dont la science « se fonde sur la tradition " ${ }^{121}$ et n'accepte aucune variante " au nom de la tradition dont il est le gardien " ${ }^{122}$. En revanche, le musicien " considère dans les mots la mesure rationnelle et le nombre, [il] se borne à exiger qu'une syllabe soit

111. Ibid., I,3.

112. Rétractations, I, 6

113. Marianne Massin, "L'esthétique augustinienne ", Laval théologique et philosophique, 61, 2005 , p. 67.

114. Michel Lemoine, "Saint Augustin et la musique " in Olivier Cullin, La place de la musique..., p. 14

115. Olivier Cullin, Brève histoire... p. 107.

116. Marianne Massin, art. cit., p. 68.

117. De musica, I, 3.

118. Anne-Isabelle Thénard-Touboulic, op. cit., p. 8.

119. Michel Lemoine, art. cit., p. 13.

120. De musica, I, 4.

121. De musica, II, 1.

122. Ibid. 
longue ou brève, selon la place que lui assignent les règles de l'harmonie $»^{123}$ par lesquelles sont associés des éléments distincts, disparates, pour composer un tout cohérent et équilibré ${ }^{124}$. Autrement dit, pour Augustin la science musicale ne semble pas être un élément figé mais une construction qui se base sur trois éléments distincts, également présents dans l'incantation magique: la mesure rationnelle, le nombre et l'harmonie. On ne peut que remarquer ici la similitude entre ces propos (opposition entre une pratique mimétique et une autre sapientielle, place primordiale de la raison, du rythme et de leur relation avec l'harmonie de la Création) et ceux manifestés par les diverses pratiques des incantations magiques (incantation populaire différenciée de celle du sage, implication du nombre dans le déroulement du rituel et dans la perception symbolique de l'univers). Cependant la théorie musicale expose une complexité qui dépasse les particularités de la pratique magique.

Le De musica aborde la musique principalement d'un point de vue technique, purement pédagogique et conceptuel, pour ensuite en nuancer les caractéristiques esthétiques, intellectuelles et religieuses ${ }^{125}$, en différenciant les rôles de la cantilène et de la jubilation.

La musique, assimilée à une quête du plaisir, est au cœur du raisonnement augustinien ${ }^{126}$. L'esthétisme augustinien, et la notion de beauté au Moyen Âge, ont fait l'objet d'études que nous ne reproduirons pas ici : soulignons néanmoins qu'il s'agit là d'un thème qui semble totalement étranger à la pratique magique et qui mérite quelques rappels.

L'appréciation morale et spirituelle de la musique, le rôle de la beauté et du plaisir, ainsi que les différentes perceptions qu'en ont les gens du peuple et les esprits éclairés, sont des éléments clés de la philosophie augustinienne qui établit une véritable gradation entre le peuple, qui possède à peine plus de raffinement et d'entendement que les bêtes, et les hommes éminents accablés par leurs lourdes responsabilités qui requièrent, on peut le supposer, un degré supplémentaire de compréhension et d'intelligence. Or, à en croire Augustin, ces hommes éclairés perdent leur supériorité morale ${ }^{127}$ en s'adonnant, même de temps en temps, aux plaisirs temporels de la musique. L'évêque explique cette position dans son traité sur le Libre Arbitre ${ }^{128}$, en affirmant que l'engouement pour les choses temporelles " peut mettre en cage qui en use mal, mais libérera celui qui (...) désirera voler comme philosophe et dépasser les plaisirs premiers et grossiers ${ }^{129}$. En réalité Augustin ne parvient pas à trancher sur l'utilisation ou le bannissement des réalisations mélodiques dans les offices religieux, car dans ses Confessions ${ }^{130}$, l'évêque rappelle comment, inspiré par Athanase, il

\section{Ibid.}

124. Edgar de Bruyne, Études d'esthétique médiévale, 2 vol., Paris, 1998, vol. 1, p. $478-479$

125. Marianne Massin, art. cit., p. 66.

126. De musica, I, IV.

127. Anne-Isabelle Thénard-Touboulic, op. cit., p. 7.

128. Saint Augustin, Du libre arbitre, II, 16.

129. Marianne Massin, art. cit., 2005, p. 64.

130. Saint Augustin, Confessions, X, 33. 
aurait souhaité dépouiller les psaumes de leur accompagnement mélodique pour les réciter uniquement avec une légère inflexion de la voix.

La lecture du texte sacré sur un fond mélodique renvoie à la cantillation, c'est-à-dire une énonciation dont la tonalité est figée ${ }^{131}$ et qui met au service de l'enseignement, de la transmission de la parole, un discours mélodique qui doit renforcer l'effet des paroles sur l'auditeur ${ }^{132}$. En dépouillant le culte religieux de toute composition instrumentale, Augustin semble bien établir que « lorsqu'il s'agit de la lecture solennelle des grands textes liturgiques (...) aucune composition musicale n'est à sa place (...) dans un acte religieux pendant lequel une transmission solennelle de certaines paroles a lieu, dans des conditions fixées dès l'origine par la nécessité puis par une tradition millénaire et qui exige des rapports mélodiques déterminés entre parole et son " ${ }^{133}$. En accord avec ce postulat, l'étude de la musique ne peut pas se limiter à une observation esthétique, elle doit permettre une élévation de l'homme pour " appréhender une plus haute réalité ${ }^{134}$ et participer ainsi à l'éducation spirituelle, à l'élévation de l'âme du croyant.

Pour comprendre la position augustinienne, il faut tenir compte du fait que les psaumes sont les textes lus les plus anciens dans la liturgie chrétienne et que la modalité de cette lecture aurait été fixée entre le $\mathrm{IV}^{\mathrm{e}}$ et le $\mathrm{V}^{\mathrm{e}}$ siècle avant Jésus-Christ ${ }^{135}$. Au moment d'organiser son propre rituel, le christianisme s'est donc largement inspiré du culte hébraïque ${ }^{136}$ mais, avec la liberté de culte apportée par l'édit de Milan, les chrétiens devaient trouver leur propre mode d'expression ${ }^{137}$ qui s'est manifesté sous la forme d'une réponse à la lecture du texte sacré ${ }^{38}$. Transformer le rythme ou la modalité de lecture revient à instaurer un nouveau rituel, une caractéristique propre qui permet au chrétien de prendre ses distances vis-à-vis des antécédents juifs, autant que des pratiques païennes.

Le mélange de la tradition grecque et de l'héritage juif a pu néanmoins déboucher sur la variété des pratiques musicales entre les Églises d'Orient et d'Occident ${ }^{139}$, mais ce sont surtout le plaisir esthétique et le danger que pouvait représenter la beauté de la musique pour le salut de l'âme qui ont été au cœur des réflexions sur la théorie musicale ${ }^{140}$. C'est ainsi que le recours aux

131. M. Hubert, "Isidore de Séville novateur? ", Revue des études latines, Société des études latines, 49 année, 1971, p. 291-292.

132. Solange Corbin, "La cantillation des rituels chrétiens ", Revue de Musicologie, n 123, 1961, p. 7.

133. Solange Corbin, art. cit., p. 3.

134. Ibid., p. 63.

135. Jacques Chailley, Histoire musicale du Moyen Âge, Paris, 1969, p. 26.

136. Ibid., p. 25.

137. Olivier Cullin, Brève histoire... p. 143.

138. Daniel Saulnier, «Le Verbe et la musique » in Olivier Cullin, La place de la musique..., p. 44.

139. Jacques Chailley, op. cit., p. 29-30.

140. Ibid., p. 37. 
instruments et au chant a pu être abordé par la discussion théologique ${ }^{141}$ et que l'Église est devenue la gardienne de l'orthodoxie et de la musique liturgique ${ }^{142}$. Le chant devient un moyen, un instrument pourrions nous dire, pour exprimer la prière et la louange divine, tant et si bien que, entre le $\mathrm{IX}^{\mathrm{e}}$ et le $\mathrm{XI}^{\mathrm{e}}$ siècles, il occupe une place prépondérante dans la liturgie ${ }^{143}$.

Or, malgré ses réticences, Augustin ne peut qu'observer les manifestations de dévotion provoquées chez le chantre par la lecture mélodique des psaumes, reconnaissant alors "la grande utilité de cette institution " ${ }^{144}$. L'évêque prend cependant soin de bien établir que le chant ne doit pas faire oublier le message du verset, car le plaisir de l'esthétique temporelle risque de surpasser le plaisir spirituel. Plus tard, dans ses Rétractations, saint Augustin rappelle comment il s'est opposé à Hilare, en défendant la récitation "à l'autel, soit avant l'oblation, soit pendant la distribution des offrandes au peuple, des hymnes tirées des psaumes " ${ }^{145}$, préservant ainsi le rôle de la parole rythmée dans la louange de Dieu. Contrairement aux préceptes magiques qui insistaient sur la manière de prononcer les incantations, la musique religieuse accorde une place plus importante à l'intensité émotionnelle du mot, par lequel les hommes transmettent ou reçoivent le message divin. Augustin fait ainsi de la musique, assimilée à une inflexion de la voix, " la forme la plus élevée de l'art, de la science et de la prière ${ }^{146}$.

Cette prise de position démontre l'évolution personnelle de l'auteur, mais elle confirme surtout que la musique peut servir à se rapprocher de Dieu, surtout lorsqu'elle est soutenue par une vive émotion qui dénote la sincérité de la dévotion. Nous retrouvons ici, une nouvelle fois, un point commun avec le rituel incantatoire qui, lui aussi, insiste sur les manifestations physiques qui expriment la volonté, sinon la dévotion et la croyance, intense du mage. Cependant, les mises en garde contre l'appât d'un esthétisme temporel, qui pourrait faire oublier la beauté de la lumière divine, ne sont pas présentes dans les grimoires. La cantillation rapproche le croyant de Dieu car elle permet un apprentissage de la parole divine, ce que ne font pas les prières des grimoires.

C'est donc dans les limites d'une quête du spirituel, d'une compréhension de la Création, que doivent être replacées les théories musicales de saint Augustin, en prenant en considération que, pour l'évêque, " la raison peut, en partant de la réalité visible qui l'entoure, s'élever par degrés jusqu'à son Créateur. Dans cette succession de degrés, les arts libéraux, rangés de façon ascendante, trouvent leur place et conduisent à la découverte des idées éternelles " ${ }^{147}$. La musique cesse d'être une simple production oisive, destinée à produire du plaisir, pour devenir elle-même un moyen de s'élever vers Dieu.

141. Ibid., p. 31.

142. Olivier Cullin, Brève histoire... p. 137.

143. Bruno Bouckaert, Mémoires du chant, Neerpelt, 2007, p. 32.

144. Confessions, X, 33.

145. Rétractations, II, 11.

146. Jean Huré, op. cit., p. 4.

147. Michel Lemoine, art. cit., p. 11-12. 
Certes, le chant religieux semble partager une même nature avec l'incantation puisque saint Augustin lui-même reconnaît que "les choses de la terre sont subordonnées aux choses du ciel, et, par une succession harmonieuse, elles associent leurs mouvements réguliers à la musique de l'univers ${ }^{148}$. Cette subordination à la musique de l'univers rappelle les propos exposés dans les grimoires et dans les théories magiques, où les actions humaines sont soumises aux qualités et aux dispositions planétaires. Mais pour Augustin la référence à une harmonie supérieure est l'occasion de dépasser le domaine de la production strictement musicale pour établir, tout au long du sixième livre du De musica, un rapport entre l'humain et le divin ${ }^{149}$.

Dans une réflexion qui anticipe les propos de al-Kindi, l'évêque affirme que le corps humain est proportionné et harmonieux car ces qualités s'observent "lorsque dans un corps deux membres forment la paire et se correspondent, ou qu'un organe unique, occupe une place intermédiaire, à une égale distance de chaque côté " ${ }^{150}$. L'homme est donc le reflet de l'harmonie universelle, "car il reçoit des nombres de plus belles proportions " tandis que son âme se laisse "épurer par les nombres divins de la sagesse ${ }^{151}$, de sorte qu'il peut l'exprimer et la recréer, ou tout au moins tenter de s'en approcher, par la modulation $\mathrm{du}$ chant, qui procure un plaisir provenant « d'une certaine mesure dans les nombres ${ }^{152}$. Bien entendu, il s'agit du plaisir de la découverte, de la perception, de l'harmonie universelle décryptée par le nombre dont les progressions ${ }^{153}$ purement arithmétiques peuvent permettre d'appréhender l'infini ${ }^{154}$. Augustin réserve un rôle de premier ordre au nombre ${ }^{155}$, dont les rapports doivent dévoiler les traces laissées par la raison divine ${ }^{156}$ et permettre ainsi de déceler les indices d'une harmonie et d'une volonté supérieure ${ }^{157}$. Il s'agit ici d'une approche plus mathématique de la symbolique numérique qui peut faire le pendant de l'approche allégorique exposée par les Cyranides.

L'approche symbolique des valeurs numériques relève de la pensée pythagoricienne $\mathrm{e}^{158}$. Par cette approche, et compte tenu de la relation établie entre la musique et le nombre, comme le souligne Béatrice Backouche, "la musique, dans le traité d'Augustin, est appelée à transcender toutes les sciences mathématiques - arithmétique, géométrie et astronomie. De fait, elle subsume toutes les autres sciences, et d'abord la première d'entre elles, qui est à la base

148. De musica, VI, 11.

149. Michel Lemoine, art. cit., p. 15.

150. De musica, VI, 13.

151. Ibid., VI, 4.

152. Ibid., II, 2.

153. Ibid., IV, 14.

154. Ibid., I, XI.

155. Anne-Isabelle Thénard-Touboulic, op. cit., p. 6.

156. Marianne Massin, art. cit., p. 65.

157. Edgar de Bruyne, op. cit., vol. 1, p. 9.

158. Béatrice Backouche, "Autour du De musica de saint Augustin ou du nombre à Dieu ", Revista de Estudios Latinos, 6, 2006, p. 84. 
des autres, à savoir l'arithmétique, la science du nombre "159. Augustin veut trouver dans chaque proportion ou grandeur numérique une valeur symbolique relative au début, au milieu et à la fin de toute création ${ }^{160}$ et pouvoir ainsi mieux se rapprocher de la vérité céleste ${ }^{161}$. C'est pourquoi, le discours musical doit s'inscrire, de la façon la plus parfaite possible, dans une structure qui reflète cette même harmonie universelle et qui ne peut échapper à la norme de l'ordre et de la mesure ${ }^{162}$. Cette implication du symbolisme numérique est également présent dans les prescriptions magiques, mais il semble s'appliquer, presque exclusivement, à la réalisation des mouvements, aux répétitions du chant comme si, loin de tenter de créer quelque chose, le mage se contentait de reproduire un système ou un modèle déjà existant. Ce rapprochement à [?] Dieu se matérialise dans le second type de production musicale défendue par saint Augustin.

Dans son serment sur le psaume 32, l'évêque rappelle qu'il est bon de louer " le Seigneur sur la harpe, [de lui chanter] des hymnes sur le psaltérion à dix cordes $»^{163}$. Cette référence s'inscrit ainsi dans une tradition où « les instruments accompagnaient volontiers les voix humaines et même exécutaient de véritables ritournelles instrumentales " ${ }^{164}$. Pourtant, l'évêque rappelle dans le même passage que les harpes ont été bannies des « saintes veilles au nom du Christ ». En réalité, Augustin interprète la forme des deux instruments, et la place de leur respective boîte de résonance, comme une allégorie des bons et des mauvais moments, dans lesquels les croyants doivent louer Dieu avec la même ferveur et intensitén ${ }^{165}$. Il associe la harpe à l'image de l'homme qui devient lui-même le véritable, l'unique, instrument de la louange divine. L'évêque met en exergue le pouvoir de l'homme à manifester sa joie et sa dévotion par le chant, qui peut alors prendre la forme de la jubilation ${ }^{166}$.

Cette aptitude est spécifiquement exposée dans le discours sur le psaume 99, où l'évêque affirme que

jubiler, ce n'est point parler, c'est exhaler sans paroles un cri de joie : c'est la voix d'une âme dont la joie est au comble, qui exhale autant que possible ce qu'elle ressent, mais ne comprenant point ce qu'elle dit dans les transports de son allégresse, l'homme après des paroles indicibles et inintelligibles exhale sa joie en cris inarticulés : en sorte que l'on comprend à la vérité sa joie dans ses cris, mais qu'il ne saurait exprimer en paroles cette joie excessive ${ }^{167}$.

159. Ibid., p. 79.

160. De musica, I, 12.

161. Ibid., V, 13.

162. Marianne Massin, art. cit., p. 63.

163. Saint Augustin, op. cit., Sermon sur le psaume XXXII, \$5-6.

164. Jean Huré, Saint Augustin musicien, Paris, 1924, p. 147.

165. Saint Augustin, op. cit., Sermon sur le psaume XXXII, $\$ 5$.

166. Jacques Chailley, op. cit., p. 27.

167. Saint Augustin, op. cit., Sermon sur le psaume XCIX, \$. 4. 
Cette fois, et contrairement à la cantilène, il ne s'agit pas de transmettre un message, mais simplement de manifester une dévotion et une allégresse intense. En exprimant la dévotion personnelle, ou communautaire, la musique se met au service de la louange divine. Son esthétisme est d'ordre divin et dépasse les appréciations physiques des mortels. Il s'agit là d'un point capital qui différencie les incantations magiques des chants religieux. La puissance de la foi et des sentiments dépasse alors la valeur intelligible du discours prononcé. La jubilation est d'ailleurs traditionnellement associée au mot Alleluia, d'origine hébraïque, et dont la signification première peut ne pas être connue par le chantre puisque le terme employé a été « le sujet d'innombrables interprétations ${ }^{168}$.

Pour ce qui est de la théorie proprement musicale et son rapport avec un certain esthétisme mystique, Augustin parle bien d'une expression inarticulée, ce qui peut être interprété comme une vocalise pouvant échapper à la "domination implacable de la parole sur la musique " ${ }^{169}$ et qui semble combler, presque en-chanter, Augustin qui ne rejette " ni l'art, s'il a une fonction anagogique, ni le beau sensible même s'il se méfie de ses attraits pour l'âme faible ${ }^{170}$. Cependant, si l'évêque défend une beauté innocente de la musique ${ }^{171}$ qui exprime une communion avec le divin, il ne minimise en aucun cas la valeur spirituelle et morale des mots prononcés. Veillant à distinguer le chant religieux des pratiques impies, le saint tient à rappeler que « notre jubilation ne ressemble point à leur jubilation, puisque notre allégresse n'a pour but que la justice, tandis qu'ils ne jubilent que dans le crime : notre allégresse est dans la confession, la leur dans la confusion ${ }^{172}$. Bien sûr, la notion de confession ici ne se rapporte pas à une confession des péchés mais à la manifestation de l'amour pour Dieu et la reconnaissance de sa gloire. Autrement dit, la jubilation religieuse est le résultat d'une expérience transcendantale, tandis que la vocalise magique voudrait justement provoquer une telle expérience.

Les textes augustiniens soulignent certaines règles de la théorie musicale qui semblent convenir au rituel incantatoire. La bonne modulation qui caractérise la science musicale, le rapport du nombre au mot qui établit un certain rythme et manifeste une harmonie universelle, l'intensité des émotions lors de la lecture du texte, peuvent être communs au chant religieux et à l'incantation. Cependant, l'apprentissage de la parole divine par le chant et les manifestations d'allégresse, produites par une révélation spirituelle, sont exclusives du chant religieux. On ne trouve pas de commentaires qui indiqueraient une extase similaire produite par l'incantation. La différence réside principalement dans

168. Gunilla Iversen « Le son de la lyre des vertus » in Olivier Cullin, La place de la musique..., p. 47.

169. Daniel Saulnier, art. cit., p. 43.

170. Marianne Massin, art. cit., p. 64.

171. Michel Lemoine, art. cit., p. 17.

172. Saint Augustin, op. cit., Discours sur le psaume XCIX, \$. 4. 
l'opposition entre l'enlèvement de l'âme de celui qui subit le charme magique, et l'élèvement de l'âme du chantre et de l'auditoire par le chant liturgique.

Il reste cependant à établir si l'harmonie universelle, manifestée par la mécanique céleste dans la pensée kindienne, est également liée aux planètes dans la théorie musicale et dans quelle mesure cette dernière relève d'une quelconque représentation astronomique et métaphysique de l'Univers. Ce sont là des questions qui ont été abordées plus tard par Boèce et Isidore de Séville.

\subsection{Boèce et la musique des sphères}

Le destin du De institutione musica de Boèce semble avoir été inégal, puisqu'il aurait été inconnu d'Isidore de Séville et, qu'après une large diffusion au XII ${ }^{\mathrm{e}}$ siècle, il aurait été moins suivi entre le XIII ${ }^{\mathrm{e}}$ et le $\mathrm{XIV}^{\mathrm{e}}$ siècle $^{173}$ pour ressurgir en Italie au $\mathrm{XV}^{\mathrm{e}}$ siècle $^{174}$.

La coïncidence de ces périodes de diffusion avec celles qui ont vu le développement des grimoires fondamentaux de la tradition magique permet d'établir un lien entre la théorie musicale boécienne et les rituels incantatoires.

Boèce commence son traité en affirmant que « d'entre les quatre disciplines mathématiques, les trois autres [arithmétique, géométrie, astronomie] œuvrent à l'exploration de la vérité, alors que la musique n'est pas seulement associée à la spéculation, mais aussi à la morale " ${ }^{175}$, en établissant ainsi que la musique dépasse la seule approche intellectuelle de la Nature, elle a trait à l'âme humaine. Boèce va développer l'aspect moral de la musique en partant du principe que " l'habileté physique répond à nos besoins comme un serviteur [et que] la raison ordonne en maîtresse ${ }^{176}$, de sorte que la "science de la musique tient bien davantage son éminence de la connaissance rationnelle que de la mise en œuvre et en acte ${ }^{177}$. Autrement dit, dans la pratique musicale, les préceptes rationnels qui structurent la production harmonique sont plus importants que la réalisation même d'une mélodie, ce qui revient, dans les grandes lignes, à reprendre la distinction augustinienne entre l'histrion et le musicien.

Or, Boèce sépare en trois catégories ceux qui pratiquent la musique. Ceux qui jouent d'un instrument sont qualifiés de serviteurs, car ils sont « exclus de la compréhension de la science musicale ${ }^{178}$, et ils ne sont pas plus musiciens que les poètes, qui «s'adonnent au chant, moins par réflexion et par raison que par un certain instinct naturel ${ }^{179}$. Le véritable musicien "soumet la science du chant

173. Christian Meyer, "Lectures et lecteurs du De Institutione Musica de Boèce au XIIIe siècle ", in Alain Galonnier, op. cit., p. 665-677

174. Christian Meyer, op. cit. p. 13

175. De institutione musica, p. 21.

176. Ibid., p. 93.

177. Ibid.

178. Ibid., p. 95 .

179. Ibid. 
non pas au service de l'œuvre, mais à l'empire de la réflexion ${ }^{180}$, il se présente en somme comme un philosophe, étymologiquement parlant, puisqu'il veut mieux comprendre les lois spécifiques et universelles qui régissent la pratique musicale. En réalité, cette triple division se résume à une distinction entre le musicien, qui connaît et étudie les lois musicales, et les autres, instrumentistes et poètes, qui en usent sans vraiment comprendre les mécanismes et les règles de la musique. Il s'agit d'une division qui correspond en tous points à la distinction faite entre l'enchanteur, qui utilise des incantations par imitation, et le sage, qui étudie les lois de la Nature et veut comprendre les sources du pouvoir incantatoire.

La musique commence ainsi à se présenter comme un moyen pour comprendre la Création ${ }^{181}$ et non comme une simple production sonore. Aussi, Boèce établit les raisons pour lesquelles cette science occupe une place privilégiée parmi les arts du quadrivium et les bases sur lesquels elle se construit. Il différencie trois types de voix qui permettent à leur tour de différencier le discours oratoire du discours musical. Le premier type de voix est utilisé pour parler ou lire un texte en prose, son énergie " est occupée à prononcer des mots et à leur donner du sens ${ }^{182}$. Le second type de voix se rapporte aux mélopées, il s'agit d' " une voix traînante réalisant un certain intervalle et, tout en mesurant les différences, elle réalise un certain intervalle, non point de silence, mais plutôt de celui d'un chant retenu et traînant ${ }^{183}$. Entre les deux se situerait la voix intermédiaire, propre à la lecture d'un poème héroïque, ne possédant « ni le débit continu de la prose, ni le mode retenu et plus nonchalant de la voix du chant ${ }^{184}$. Cette triple division ne fait que confirmer l'idée d'àpropos de la production musicale sur laquelle insistait saint Augustin, mais elle apporte surtout un point de vue nouveau sur la modalité de lecture des textes incantatoires. Les textes des prières magiques se présentent soit en prose soit sous forme de mots isolés, ils ne sauraient donc être interprétés, ou lus, avec les mêmes dispositions que requièrent les poèmes héroïques. En outre, leur désignation en tant que prière semble bien les distinguer de ces textes en prose qui requièrent une voix continue. Le ton et le rythme de la mélopée semblent donc correspondre le mieux au texte incantatoire, sans pour autant lui conférer un pouvoir particulier. Sur ce dernier point, Boèce propose une approche à peine évoquée par saint Augustin.

En se fondant sur les effets que produisent les tambours et les trompettes sur les guerriers ou encore les rythmes langoureux qui accompagnent certaines danses lascives, et à l'écoute desquels " l'esprit des auditeurs (...) se disperse peu à peu et ne conserve aucune trace de ce qui est honnête et juste " ${ }^{185}$, Boèce conclut que la musique touche naturellement les hommes. Cette capacité est

180. Ibid., p. 93.

181. Christian Meyer, "Lectures... ", p. 668.

182. De institutione musica, p. 53.

183. Ibid.

184. Ibid.

185. Ibid., p. 23. 
rendue possible selon lui par une consonance entre l'agencement harmonieux propre à l'homme et celui des sons ${ }^{186}$. Alors que saint Augustin établit l'harmonie du corps humain en se référant aux proportions numériques, Boèce se réfère surtout à " l'ensemble formé par notre âme et notre corps [qui] est organisé selon une structure musicale. De même, en effet, que les sensations du corps affectent celui-ci, les pulsations du cœur sont provoquées par les émotions ${ }^{187}$. L'âme émue fait réagir le corps, et les sensations perçues par ce dernier peuvent l'affecter en retour ${ }^{188}$. Cette double influence expliquerait pourquoi la musique apaise les mœurs. Pour étayer sa thèse, Boèce rapporte alors deux histoires dans lesquelles Pythagore et Empédocle auraient su calmer la colère et les envies meurtrières de leurs semblables par la seule énonciation de vers mélodieux au rythme posé ${ }^{189}$. La capacité propre à la parole mesurée, bien modulée dirait saint Augustin, ainsi qu'à certaines compositions mélodiques ${ }^{190}$, d'influencer le comportement d'autrui, repose dans sa faculté à renforcer ou faire rejaillir l'harmonie de chaque être. On l'aura compris, cette réflexion ne contredit pas le point de vue de al-Kindi et de Roger Bacon, pour qui les voix, les sons, auraient le pouvoir de renforcer ou d'entraver les influences planétaires et les comportements de l'environnement. Cependant, la modulation, la diction rythmée du mot, appartient au domaine de la production externe de la voix. Or, dans la théorie magique, telle qu'elle est présentée par al-Kindi, le mot lui-même possède un pouvoir propre qui lui permet d'agir sur le monde environnant. Il s'agit donc de trouver, d'après les lois musicales, la source de cette faculté, en distinguant les différents types de musique.

Boèce distingue justement trois genres de musique, "la première est la musique du monde, la deuxième est la musique de l'homme, la troisième est celle qui est réalisée sur certains instruments (...) qui sont au service de la cantilène ${ }^{191}$. La musique du monde est celle qui manifeste la structure de la Création puisqu' " on l'observe surtout dans le ciel lui-même, dans la combinaison des éléments ou dans la diversité des saisons ${ }^{192}$. Elle est produite par le mouvement des cieux car " le mouvement, si rapide, des grands corps, ne saurait produire nul son, d'autant plus que les courses des étoiles sont unies par une cohésion d'une harmonie telle qu'il ne soit possible de concevoir rien d'aussi parfaitement ajointé, ni d'aussi uni ${ }^{193}$. La musique des cieux est une conséquence de leur unicité, elle manifeste l'harmonie de tout ce qui existe,

186. Ibid.

187. Ibid., p. 29.

188. Ibid., p. 27.

189. Ibid., p. 27-29.

190. La guérison de Saül par David jouant de la harpe (I sam. 16, 23) ainsi que les commentaires d'Isidore de Séville sur les débuts de la musicothérapie (Etimologías, IV, 13, 3) s'inscrivent dans cette optique. La musique se présente comme un agent pouvant toucher l'âme de chaque individu.

191. De institutione musica, p. 32-33.

192. Ibid., p. 33.

193. Ibid. 
elle serait donc créatrice et bénéfique. Or, Boèce parle bien de sons émis par les planètes, produits par leur propre mouvement. Â ce sujet, il faut rappeler que, d'après une définition qui ne sera jamais mise en doute ${ }^{194}$, la nature des sons est strictement vibratoire, elle est produite par un choc, "et il ne saurait en aucune manière y avoir vibration ni choc sans qu'un mouvement ait précédé " ${ }^{195}$. Le mouvement provoque les vibrations et les vibrations des sons. Chaque mouvement particulier provoque un son particulier et donc chaque planète, à la vitesse et aux proportions propres, produit un son défini, exception faite de la Terre, censée demeurer immobile et donc, silencieuse. Les mouvements les plus rapides, ceux des planètes les plus éloignées, produisent des sons aigus, les planètes les plus proches, dont la Lune, au mouvement plus lent, émettent des sons graves ${ }^{196}$. Cette distinction sonore, construite sur une différence vibratoire propre à chaque astre peut concorder avec la théorie kindienne des rayons, dont les différentes natures sont la conséquence des différentes qualités et personnalités des astres. La seule différence remarquable est que si pour al-Kindi le son émet des rayons, des vibrations, comme tout agent en action, la théorie musicale considère le son comme une conséquence de l'action réalisée par l'agent en mouvement.

Bien entendu, cette approche astronomique de la musique est directement inspirée des écrits platoniciens, et tout particulièrement du Timée, que Boèce a exploité dans son De Institutione Arithmetica, pour y montrer que "la composition du corps et de l'âme du monde obéit à des lois arithmétiques »197 des lois qui sont également présentes dans la perception de la musique ${ }^{198}$. Sous l'influence de la pensée aristotélicienne ${ }^{199}$, qui affirme que les planètes se meuvent en silence et par conséquent ne peuvent produire aucune musique audible ou sonore ${ }^{200}$, la théorie de la musique des sphères a été peu à peu abandonnée $e^{201}$, ce qui ne signifie pas que l'influence des astres sur les hommes ait été définitivement oubliée ou écartée.

Albert le Grand est le meilleur exemple de cette double approche des influences stellaires puisque dans le second livre de son De Celo et Mundo, il refuse l'idée d'une musique sonore des sphères ${ }^{202}$, pour ne retenir qu'une musicalité harmonique, construite sur l'idée de proportions et de valeurs arithmétiques ${ }^{203}$, plus que sur l'émission de tonalités mélodiques. Pourtant,

194. Christian Meyer, « Lecture... », p. 667.

195. De institutione musica, p. 35.

196. Ibid., p. 85.

197. Béatrice Bakouche, «Boèce et le Timée » in Alain Galonnier, op. cit., p. 10.

198. Ibid.

199. Dominique Proust, L'harmonie des sphères, Paris, 2001, p. 50; Christian Meyer, "Lecture... ", p. 666.

200. Aristote, De Caelo, édition de Jean-Pierre Verdet, Paris, 1993, II, 9.

201. Chirstian Meyer, art. cit., p. 121-122.

202. Albert le Grand, De Celo et mundo, édition de P. Hossfeld, Cologne 1971, II, 2, 10.

203. Ibid., p. 163. 
il continue de défendre une similitude entre l'homme et le ciel, entre le microcosme et le macrocosme $e^{204}$, qui favorise l'influence des figures astrales sur la vie des hommes.

Dans son Monde Minéral, Albert le Grand part du principe que "les configurations (figuras) du ciel sont les premières et viennent avant celles de tout ce qui a été engendré par la nature et par les arts. En effet, ce qui vient en premier, en genre et en ordre, parmi les forces génératrices sans aucun doute impose sa causalité à tout ce qui vient après, d'une façon appropriée à chaque cas $»^{205}$. Par cette influence primordiale, qui précède et qui lie finalement toutes les choses faites par la nature et par les arts, le bénédictin conclut que " la configuration du ciel aura donc une causalité dans toute figure engendrée par la nature, car l'art se fond dans l'origine de la nature. En effet, selon ce que nous avons dit, l'origine de l'art est la nature telle qu'elle résulte de son origine céleste qui est une intelligence active, de même que l'intelligence est à l'origine de l'art ${ }^{206}$. Albert le Grand défend donc l'idée d'une harmonie céleste, qui abandonne l'existence des sons ou des tons planétaires, mais qui s'inscrit dans la théorie musicale en conservant le rapport des proportions et de la concordance des mouvements qui existent dans la Création. Autrement dit, si la musique des astres est abandonnée, la conviction en une harmonie céleste demeure le référent primordial pour toute réflexion d'ordre spirituel, scientifique ou métaphysique ${ }^{207}$.

Or, comme le souligne Christian Meyer, les références aux lois qui soutenaient une musique sonore des sphères sont toujours présentes dans les écrits de Michel $\mathrm{S} \mathrm{cot}^{208}$, qui fait toujours correspondre une note à chaque planète ${ }^{209}$. De même le Tratado de musica, plus tardif, continue à reproduire la notation musicale par le moyen de lettres ${ }^{210}$, en utilisant un système qui rappelle la nomenclature ptolémaïque d'identification des planètes ${ }^{211}$, tout comme il perpétue le souvenir d'une musique céleste harmonique ${ }^{212}$.

Cette survie des anciennes théories démontre que l'arrivée de nouvelles idées, voire la confirmation de nouvelles découvertes et de nouvelles percées dans la compréhension des lois naturelles, ne font pas disparaître les anciennes croyances. La survie de l'astrologie, malgré le développement scientifique de l'astronomie, permet de constater que les croyances dépassées ont tendance à se réfugier dans des domaines plus restreints, souvent liés à l'irrationnel de l'immense majorité du peuple comme l'évoquait Augustin. La magie fait partie de ces domaines et elle offre un refuge aux théories de la musique céleste,

204. Ibid., p. 5.

205. Albert Le Grand, Le monde minéral, édition de Michel Angel, Paris, 1995, p. 374.

206. Ibid., p. 375.

207. Weill Parrot, op. cit., p. 268-269.

208. Christian Meyer, art. cit., p. 122.

209. Ibid., p. 124-125.

210. Tratado de musica, fols. $2 \mathrm{v}^{\circ}-2 \mathrm{r}^{\circ}$

211. Jacques Chailley, op. cit., p. 41-46.

212. Tratado de musica, fol. $7 \mathrm{v}^{\circ}$. 
fondées sur une vision homocentrique fausse, alors que la théorie musicale les abandonne progressivement.

Il est certain que l'attribution des notes aux planètes a subi au cours des siècles des nuances, et parfois des modifications dans l'ordre des notes et des tonalités ${ }^{213}$. Il demeure cependant une constante qui lie chaque planète à une note et à un jour ${ }^{214}$, plaçant chaque moment de la journée et chaque action humaine sous le sceau d'une note musicale particulière. On peut donc admettre que si les notes et les planètes agissent de concert ou par opposition, si chaque planète possède sa note et son jour, si chaque discours, textuel ou musical, requiert une modalité d'énonciation particulière et appropriée au but du discours émis, le mage doit alors tenir compte de tous ces éléments pour réaliser une incantation qui soit efficace. Lorsque les grimoires expliquent qu'une opération magique doit être réalisée sous l'influence d'un astre en particulier, ils sont aussi en train d'indiquer que la prière magique, qui peut adopter le rythme de la mélopée, et surtout les noms isolés des esprits planétaires doivent être prononcés sur une tonalité spécifique, celle qui correspond à l'esprit planétaire que le mage veut conjurer. Pour cela il doit suivre un modèle ou se rattacher à une tradition que les auteurs jusqu'ici cités ne mentionnent pas, mais qu'Isidore de Séville présente dans ses Étymologies.

\subsection{Isidore et la syrinx}

Dans les Étymologies, Isidore de Séville ouvre son chapitre sur la musique par des propos qui rappellent ceux de saint Augustin. Pour lui, la musique est la maîtrise de la modulation du son, exprimé par le chant ${ }^{215}$. Il identifie l'art de la modulation selon les schémas métriques et les chants ${ }^{216}$, et il reprend par conséquent la vision prosodique de saint Augustin. Cependant, le sévillan n'insiste pas, comme l'évêque d'Hippone, sur la différence essentielle entre la modulation et la bonne modulation.

D'un point de vue conceptuel, Isidore établit la triple division de l'harmonie, du rythme et de la métrique ${ }^{217}$, qui correspondent aux sons, aux paroles et aux mètres, dans la tradition augustinienne de la théorie musicale. Isidore distingue également les musiques harmonique, organique et rythmique ${ }^{218}$ qui correspondent à la voix, au souffle et à la pulsion/percussion. Cette triple division coïncide avec les théories musicales déjà établies par Boèce, définissant les différences entre une musique céleste, humaine et instrumentale.

213. Dominique Proust, op. cit., p. 79-84.

214. Jacques Chailley, op. cit., p. 22.

215. Etimologias, III, 15, 1 : «Musica est peritia modulationis sono cantuque consistens ».

216. Ibid., I. 2,2.

217. Ibid., III, 18.

218. Ibid., III, 19. 
Dans un classement traditionnel des arts libéraux, la musique isidorienne est subordonnée à l'arithmétique ${ }^{219}$, non parce qu'elle est issue du calcul des nombres, mais tout simplement parce que l'arithmétique serait le seul art à exister par lui-même ${ }^{220}$. Isidore explique d'ailleurs que le calcul de la moyenne musicale se fait selon les rapports arithmétiques et géométriques ${ }^{221}$ et se rapproche ainsi de la vision pythagoricienne de la musique fondée sur le rapport des nombres tel que le décrit Boèce. Ainsi, la musique isidorienne appartient à la philosophie spéculative doctrinale ${ }^{222}$, au même titre que l'arithmétique, la géométrie et l'astronomie, mais elle se distingue des autres pratiques mathématiques car elle porte sur les nombres liés aux sons ${ }^{223}$. Autrement dit, la musique se présente toujours comme une expression de valeurs numériques qui peuvent transcrire l'harmonie de la Création. Cette capacité s'explique, d'après Isidore, parce que la Création elle-même aurait été réalisée par la concordance des sons qui confèrent à la mécanique céleste son harmonie ${ }^{224}$. Pour cela Isidore affirme que rien ne peut être parfait sans la musique, car elle est perçue comme un agent qui manifeste l'essence même de tout ce qui existe.

Cette capacité est exprimée par l'évocation du dieu Pan et de sa syrinx. Isidore de Séville explique que les sept chalumeaux de l'instrument correspondent aux sept planètes qui régissent les différents cieux, respectivement liés à sept notes et à sept voix ${ }^{225}$. Les traits physiques du dieu païen sont comparés aux différentes manifestations sidérales, du Soleil, de la Lune ou des étoiles. Il est aussi une manifestation de la Nature sauvage, de la force de la terre et de la puissance des vents. Loin d'être une image grotesque ou infernale, il représente le Tout en harmonie, autrement dit un véritable Cosmos. Le sévillan insiste alors pour souligner que l'instrument du dieu Pan est une figuration de l'harmonie céleste.

La référence à une divinité mythologique pour représenter cette harmonie est d'autant plus importante qu'elle peut renvoyer à d'autres personnages du panthéon païen.

La syrinx, avec la flûte et la lyre, traditionnellement associées à Pan, Hermès et Athéna ${ }^{226}$, composent la triade instrumentale qui traduit " l'image de la musique cosmique ${ }^{227}$. Ces divinités, transformées en esprits planétaires dans la conception magique de l'univers, jouent des rôles prépondérants dans les opérations magiques. Alors que Pan serait le protecteur de la divination et Athéna/Minerve la protectrice de la richesse et de la sagesse, Hermès/Mercure est le messager des dieux, vainqueur des forces infernales et pourvoyeur de

219.Ibid., III, 2.

220. Ibid., III, 1, 1.

221. Ibid., III, 8.

222. Ibid., II, 24, 10.

223. Ibid., II, 24, 15.

224. Ibid., III, 17, 1.

225. Ibid., VIII, 11, 82-83.

226. Jean Marie Fritz, art. cit., p. 24.

227. Ibid., p. 37. 
connaissances. Leurs instruments peuvent donc être assimilés à des clefs qui ouvrent les portes du Ciel. Par leur intermédiaire, la musique deviendrait une manifestation audible pour l'homme de la personnalité de ces puissances cosmiques, tout comme l'utilisation de ces instruments signifierait pour le musicien, ou pour le mage, un rapprochement avec les forces naturelles et surhumaines. La référence isidorienne à Pan établit un lien entre la théorie musicale appartenant aux arts libéraux et la pratique incantatoire. Les deux approches partagent les mêmes sources et souvent les mêmes référents pour exprimer leur propre déroulement. Le saint sévillan n'exploite pas davantage cette image, mais son discours, est teinté d'une théorie gnostique, présente dans les hymnes orphiques ${ }^{228}$ inspirés par le pythagorisme ${ }^{229}$ et le stoïcisme ${ }^{230}$, qui défend la pratique de la vocalise liée aux sept planètes ${ }^{231}$.

L'évêque de Séville renoue ainsi avec l'idée d'une musique sidérale. Plus important encore, en associant chaque note, et donc chaque roseau de la syrinx à une planète, Isidore donne une réalité matérielle, avec une longueur et un diamètre mesurables, propres à chaque chalumeau, aux tonalités qui jusque là ne pouvaient être retenues que par la mémoire. Cette limitation de la production musicale est confirmée par le sévillan lorsqu'il associe la musique aux Muses, filles de Jupiter et de Mémoire, soulignant ainsi la nature éphémère de la composition musicale qui dépend du souvenir des hommes. D'après Isidore, les chants de l'Antiquité se seraient uniquement transmis par voie orale, et reposeraient exclusivement sur la mémoire des hommes ${ }^{232}$ car, pour le sévillan, la transcription des notes est impossible ${ }^{233}$. Il est certain que, le système actuellement en vigueur n'ayant été établi qu'au XIX ${ }^{\mathrm{e}}$ siècle, tout musicien ou musicologue s'est trouvé confronté à la difficulté de transcrire le discours musical en utilisant des signes graphiques, qui peuvent relever d'une invention personnelle ${ }^{234}$. Isidore, conscient du "rôle pratique que pouvait jouer la ponctuation dans la psalmodie (...) ainsi que dans la lecture liturgique des textes sacrés ${ }^{235}$, se serait lui-même essayé à composer un système de notation rythmique. Dans ses Étymologies, il consacre en effet quatre chapitres aux syllabes, aux mètres, aux accents et, surtout, aux signes qui transcrivent l'accentuation (figuris accentuum) ${ }^{236}$, en s'inscrivant ainsi dans une tradition qui cherche à déterminer « les bonnes coupures à faire dans la lecture d'un texte,

228. Miguel Periago Lorente, op. cit., p. 68-69.

229. Ibid., p. 65.

230. G. Roca-Serra, "Le symbolisme du dieu Pan chez Isidore de Séville », Revue du Moyen Âge Latin, Lyon, 1945, p. 6

231. Jacques Chailley, op. cit., p. 27.

232. Étymologies, III, 15, 2.

233. Ibid.

234. Georges Jean, L'écriture, mémoire des hommes, Paris, 1987, p. 152.

235. Jacques Fontaine, Isidore de Séville et la culture classique dans l'Espagne wisigothique, Turnhout, 1983 , p. 73.

236. Étymologies, I, 16-19. 
mais aussi, et surtout, le type de mélodie dont il fallait orner et préparer ces coupures dans les proclamations liturgiques ${ }^{237}$.

Il s'agit ici d'une nouvelle problématique qui dépasse le but de ces quelques pages, puisqu'elle implique l'analyse de l'écriture de la musique ${ }^{238}$, et du rythme du discours prononcé, au moyen d'un système où « le signe écrit (...) ne renvoie pas immédiatement à la chose signifiée sinon en le faisant par la médiation de la pensée ${ }^{239}$. Pour l'heure, on peut seulement signaler que, si les incantations magiques nécessitent une diction cadencée et normée, elles ne se distinguent pas des autres textes lus. Elles requièrent pour cela de bonnes coupures et une hauteur de voix spécifique, qui pourraient être indiquées par les différents caractères magiques qui accompagnent souvent ces textes.

Les écrits isidoriens établissent le lien direct entre la théorie musicale stricte, basée sur une conception de la modulation et un calcul mathématique où la symbolique des nombres tient une place importante, avec une tradition mystique dans laquelle puisent abondamment les pratiques magiques. Dès lors, si les notes musicales, qui permettent de créer le discours mélodique, autant que le symbolisme numérique, qui donne une valeur spécifique aux structures d'énonciation musicale, sont communes aux traditions magiques et sapientielles ou religieuses de la pratique musicale, il est possible de conclure que les incantations magiques suivaient les mêmes règles d'énonciation et de production que le chant ou la mélodie religieuse.

\section{Conclusion}

L'influence des astres sur les opérations incantatoires, dans la théorie magique et astrologique, est l'une des prémisses fondamentales dans tous les grimoires. Cependant, l'influence de la théorie musicale dans les opérations magiques a été quelque peu négligée, voire écartée.

Puisque les différents grimoires préconisent l'étude des arts libéraux, et surtout du quadrivium, il semble plus que probable que le mage auquel s'adressent de tels écrits connaît les règles de la pratique musicale. En outre, dans ces mêmes écrits, l'identification des incantations à des prières établit une relation formelle entre les discours magique et religieux, sinon dans le fond au moins dans leur forme. Un dernier élément à tenir en compte est le respect impératif d'un rituel strict, pré-établi, aussi bien dans la diction que dans la gestuelle ou la préparation des éléments qui participent à l'opération magique. La diction doit donc être soumise à une norme connue du mage.

Le pouvoir des talismans réside dans les rayonnements des étoiles capturés par le mage autant que dans l'intensité de ces émotions et la foi qu'il porte au succès de son opération. L'enchanteur prononce également des mots spécifiques

237. M. Hubert, art. cit., p. 290.

238. Olivier Cullin, «L'œil et l'esprit » in Olivier Cullin, op. cit., p. 94-95.

239. Ibid., p. 91. 
pour parfaire son œuvre. Il peut se limiter à répéter un certain nombre de fois un chapelet de noms, ou à prononcer un long discours dont le registre va de l'imprécation à la supplication. Alors que la puissance du discours peut provenir de la structure même des phrases, le pouvoir des mots isolés ne peut provenir que des vibrations que les paroles et les voix humaines sont censées produire. Il n'en reste pas moins nécessaire de les prononcer correctement, avec le bon rythme et la bonne hauteur de voix.

C'est dans cette réalisation pratique qu'interviennent les différents éléments de la théorie musicale. L'association du rythme à la cadence et à la hauteur de voix s'inscrit dans l'identification de la musique à une bonne modulation qui ne doit pas faire oublier le sens du texte, mais qui peut permettre un rapprochement avec le divin en exprimant l'intensité de la volonté ou de la dévotion de l'orateur. Ce sont là des éléments qui peuvent convenir aux chants religieux et magiques. Leur seule différence réside dans l'expression esthétique qu'ils peuvent véhiculer. Il s'agit d'un rapport à la beauté qui peut représenter un certain danger pour le chantre religieux mais qui est formellement absent dans le chant incantatoire.

$\mathrm{Au}$ respect de la bonne modulation vient ensuite s'ajouter la nature même des notes et des sons émis. Inspiré par une astronomie musicale, où chaque astre possède une personnalité et produit un son spécifique dans son déplacement, la théorie musicale a établi un rapport direct entre les gammes et les rapports numériques issus de l'école pythagoricienne, et les planètes. La musique devient ainsi une manifestation sonore de l'harmonie céleste. Dès lors, les instruments se transforment en outils pour recréer cette harmonie céleste en reproduisant, dans le monde humain, les sons des mondes sidéraux. La production mélodique établit alors un lien entre le monde des hommes et celui des esprits planétaires.

L'évolution de la connaissance astronomique a pu faire évoluer la théorie musicale qui a finalement abandonné la conception d'une musique des sphères. Cependant, cette même évolution n'est pas parvenue à modifier les théories des pratiques magiques. Celles-ci, toujours fondées sur les préceptes des premiers grimoires, continuent d'accepter une influence directe des différentes planètes sur la destinée humaine. Les astres sont considérés comme des agents qui produisent des effets sur l'ensemble de la Création. Aussi, les grimoires préconisent d'agir en harmonie avec la planète tutélaire pour réaliser une incantation ou un talisman, en réalisant le rituel au bon moment avec des objets aux couleurs de l'esprit planétaire invoqué et en prononçant correctement les mots indiqués. Or, compte tenu de l'association tonale de chaque planète, il serait impossible pour le mage de s'adresser à un esprit planétaire sur un ton qui ne lui correspond pas. Le choix d'une tonalité pour l'énonciation de l'incantation devait être prédéfini par l'esprit planétaire auquel s'adressait celleci. Le mage devait donc être instruit dans la science musicale autant que dans l'astronomie.

Il n'en reste pas moins à déterminer le rythme de l'énonciation, car si la définition boécienne de la mélopée peut s'accorder au discours incantatoire, elle n'explique pas réellement où et comment réaliser les coupures nécessaires 
pour la bonne énonciation. Il en résulte que le discours incantatoire, inscrit maintenant dans une tradition musicale établie par le quadrivium, connaît le même problème que la production musicale classique, ne possédant pas de véritable système d'écriture pour transmettre les modalités d'énonciation. Il pourrait ainsi s'accorder une fois de plus avec les propos isidoriens sur le rôle de la mémoire et de la répétition. Cependant, compte tenu des efforts réalisés pour transcrire les mélodies, il est possible que la production magique se soit également essayée à une telle démarche, ce qui requiert des recherches spécifiques dans le domaine de l'écriture et des symboles magiques. 